This document is published in:

Games and Economic Behavior (2009), 65(2), 372-405.

DOI: 10.1016/j.geb.2007.10.015

(C) 2008 Elsevier Inc. 


\title{
Information acquisition in auctions: Sealed bids vs. open bids
}

\author{
Ángel Hernando-Veciana* \\ Departamento de Economía, Universidad Carlos III, 28903 Madrid, Spain
}

\begin{abstract}
This paper studies the incentives of a bidder to acquire information in an auction when her information acquisition decision is observed by the other bidders before bidding. Our results show that the sealed bid (second price) auction may induce more information acquisition about a common component of the value than the open (English) auction but less about the private component of the value. Moreover, we show that more information about the private value and less information about the common value may improve efficiency and revenue. Consequently, our results suggest new arguments in favor of the open auction.
\end{abstract}

JEL classification: D41; D44; D82

Keywords: Auctions; Open information acquisition; Asymmetric information

\section{Introduction}

Most auction theory models assume that bidders have some private information. However, relatively little is known about the incentives of bidders to acquire it. This question is also a practical auction design problem since bidders' private information acquisition affects the efficiency in the auction and the auctioneer's expected revenue.

In this paper, we study a bidder's incentives to acquire information about the object for sale when the information acquisition decision is observed by the other bidders and so can affect

* Fax: + 34916249329 .

E-mail address: angel.hernando@uc3m.es.

URL: http://www.eco.uc3m.es/ahernando/. 
their bids. An example is oil-tract auctions in which information is basically acquired through exploratory drills which are easily visible. ${ }^{1}$

In particular, we compare the incentives to acquire information in a (second price) sealed bid auction and in an open (English) auction. In both formats, the winner is the bidder with highest bid and the price the highest losing bid, but they differ in that the first auction is static whereas the second one is dynamic. In this sense, our paper contributes to the comparison between static and dynamic auctions.

In our framework, bidders' values have common and private components. Our results show that if the information acquisition is about the former, the sealed bid auction induces more information acquisition than the open auction, whereas the order is the opposite, at least if there are sufficiently many bidders, when the information acquisition is about the bidder's private value. We show that these results imply new arguments why the open auction may give greater expected revenue and be more efficient than the sealed bid auction.

We argue that the difference in incentives in our two auctions is explained by the fact that bidders can track the bidding of the bidder who acquires information in the open auction but not in the sealed bid auction.

There are relatively few models of observable information acquisition in auctions. Milgrom and Weber (1982b) studies whether the value of acquiring information in a first price auction is greater when it is observed by the other bidders. Although we do not study this question our analysis suggests other forces operating in our two auction formats. For instance, private information acquisition in our model does not decrease the other bidders' bids as in their model. Milgrom (1981), Engelbrecht-Wiggans et al. (1983), and Hernando-Veciana (2004) derive some partial results as a by-product, and Larson (2004) and Hernando-Veciana and Tröge (2005) focus on the value of private information. The last two papers differ from ours in that they are restricted to the open auction and to information acquisition about the common value, whereas we also study the sealed bid auction and information acquisition about the private value.

One technical difficulty in the analysis of observable information acquisition is that one must study auction games in which bidders are asymmetric. ${ }^{2}$ In this respect, we propose a tractable model in which bidders differ in the quality of their private information. Other similar models are those by Hernando-Veciana (2004), Larson (2004) and Hernando-Veciana and Tröge (2005) which have been referred to previously, and the model of an independent and simultaneous paper by Boone and Goeree (2005). This last paper differs from ours in that it studies the optimal auction design and does not consider the question of information acquisition. Parreiras (2002) provides an alternative model of asymmetric common value auctions.

Other papers have studied covert information acquisition; two early examples are Matthews (1984) and Lee (1985), and more recently, Hausch and Li (1993), Persico (2000), Bergemann and Välimäki (2002), and Hagedorn (2004). While none of them addresses the comparison of our two auction formats, an application of the techniques of Persico (2000) may be used to prove that the sealed bid auction gives greater incentives to acquire information than the open auction.

\footnotetext{
1 The study of observable information acquisition is also useful to analyze whether a bidder has incentives to make it public her acquisition of information, or whether the auctioneer wants to reveal who acquired information in case she knew it. This is the case, for instance, when an authorization of the auctioneer is required to acquire information.

2 Other branch of the literature, which includes McAfee and McMillan (1987), Harstad (1990), Levin and Smith (1994), Stegeman (1996), Engelbrecht-Wiggans (2001) and Ye (2007), assumes that a bidder must incur in some information acquisition costs to enter the auction. Note that this alternative model of information acquisition does not have the aforementioned difficulties since all the bidders who enter the auction are ex ante symmetric.
} 
The intuition is that a bidder can infer in the equilibrium of the open auction the signals of the other bidders from their bids, which gives her information about her own signal if signals are correlated, and thus decreases her incentives to acquire it. We assume that private information is independent across bidders to eliminate this effect, so that we can focus on the implications of the observability of the information acquisition decision.

Compte and Jehiel (2002) also compare information acquisition in the sealed bid auction and the open auction. However, their paper differs from ours in that they study a pure private value model. Under this assumption it is irrelevant whether the information acquisition choices are observed since they do not affect the other bidders' bids, at least in the auction formats that Compte and Jehiel (2002) study.

The rest of the paper is organized as follows. The next section provides the assumptions of the model. Basically, we study a two-stage game: first, an information acquisition stage, and second, an auction game. We study the second stage in Section 3, and the first stage in Section 4. In Section 5, we study the implications of our results for the efficiency of the auction and the auctioneer's expected revenue. Finally, Section 6 offers our conclusions. We also include three appendices, the first one with a discussion of our notion of more informative signals, the second one with all the proofs and the third one with an example that illustrates some of our assumptions.

\section{The model}

One unit of an indivisible object is put up for sale to a set $I \equiv\{1,2, \ldots, n\}$, where $n \geqslant 3,{ }^{3}$ of risk neutral bidders. A generic Bidder $i \in I$ puts a monetary value $T_{i}+\sum_{j \in I} Q_{j}$ on the consumption of the good, where $T_{i}$ (for taste) is a private value and $Q_{i}$ (for quality) a common value component. Additive separability, although restrictive, simplifies our analysis by allowing the application of Myerson's (1981) techniques.

Bidders play a two stage game. In the first stage, each bidder chooses simultaneously ${ }^{4}$ and independently a signal from a family $\left\{X_{i}^{\eta}\right\}_{\eta \in[0,1]}$ at a monetary $\operatorname{cost} c(\eta) \geqslant 0$ where $c^{\prime}(\eta)>0$ for $\eta>0$. The bidders' choices are made public at the end of the first stage. We assume $\left(T_{i}, Q_{i},\left(X_{i}^{\eta}\right)_{\eta \in[0,1]}\right)$ to be a random vector independent and identically distributed across bidders. We also assume that $E\left[V_{i} \mid X_{i}^{\eta}=x\right]$ is a twice continuously differentiable function of $x$ and $\eta$, where $V_{i}$ denotes here and in what follows an element of $\left\{T_{i}, Q_{i}\right\}$.

In the second stage, each bidder first observes privately the realization of the signal she chose in the first stage and, second, bids in an auction. We consider two auction formats: an open auction and a sealed bid auction which are described respectively in Sections 3.1 and 3.2.

We assume some structure in our family of bidders' signals: first, that the marginal distribution of $X_{i}^{\eta}$ is uniform on $[0,1]$; second, that $\frac{\partial E\left[V_{i} \mid X_{i}^{\eta}=x\right]}{\partial x}>0$, for $x \in[0,1]$ and for both $V_{i}=T_{i}$ and $V_{i}=Q_{i}$; and third, one of the following two alternative assumptions:

3 We assume that $n \geqslant 3$ since our open and sealed bid auctions are strategically equivalent for $n=2$.

4 The simultaneity of the bidders' information acquisition decision may seem in conflict with the assumption that the information acquisition decisions are observable before the auction game. One justification is that bidders information acquisition decision must be taken before the visible part of the process of information acquisition starts. For instance, an oil company may need permissions, hire the equipment and contract consulting services before starting a drill. A more basic reason for this assumption is that we want to focus on the incentives to acquire information abstracting from other strategic issues in the information acquisition game like first mover advantages, predatory strategies and endogenous timing. They seem relevant issues but more appropriate for extensions. 
- The Q-information assumption: $\frac{\partial^{2} E\left[Q_{i} \mid X_{i}^{\eta}=x\right]}{\partial \eta \partial x}>0$ and $\frac{\partial^{2} E\left[T_{i} \mid X_{i}^{\eta}=x\right]}{\partial \eta \partial x}=0$, for any $x, \eta \in[0,1]$.

- The T-information assumption: $\frac{\partial^{2} E\left[T_{i} \mid X_{i}^{\eta}=x\right]}{\partial \eta \partial x}>0$ and $\frac{\partial^{2} E\left[Q_{i} \mid X_{i}^{\eta}=x\right]}{\partial \eta \partial x}=0$, for any $x, \eta \in[0,1]$.

We explain in Appendix A.1 using the arguments of Athey and Levin (2001) that the first postulate is simply a normalization, that the second one ensures an increasing best response in the auction games, and that the third postulate orders the family of signals $\left\{X_{i}^{\eta}\right\}_{\eta \in[0,1]}$ in the sense of more informative signals about the common value, if we make the first assumption, and about the private value, if we make the second assumption. In general, we shall refer to $\eta$ as the bidder's information precision. We discuss in the Conclusions how our distinction between information acquisition about the private and the common value relates to other models of information acquisition in auctions. Note that our second assumption above also plays another role. It rules out the possibility of either a pure common value model or a pure private value model.

Finally, let $\mu(x, \eta) \equiv E\left[Q_{i} \mid X_{i}^{\eta}=x\right]-E\left[Q_{i} \mid X_{i}^{\eta} \leqslant x\right]$ and assume it to be increasing in $x$, for any $\eta \in[0,1]$. $^{5}$

The following example illustrates all our assumptions:

Example A. Let $Q_{i}, T_{i}, U_{i}$ be three random variables independent and identically distributed according to a uniform distribution in $[0,1]$. Let $X_{i}^{\eta}$ be a lottery equal to $Q_{i}$ with probability $\eta_{T}(\eta)>0$, equal to $T_{i}$ with probability $\eta_{Q}(\eta)>0$, and with the remaining probability, equal to $U_{i}$. Suppose that $\eta_{T}$ and $\eta_{Q}$ are differentiable and $\eta_{T}^{\prime}(\eta)=0$ and $\eta_{Q}^{\prime}(\eta)>0$ under the Q-information assumption, and vice versa under the T-information assumption. To see that it satisfies all our assumptions note that, $E\left[Q_{i} \mid X^{\eta}=x\right]=\eta_{Q}(\eta) x+\left(1-\eta_{Q}(\eta)\right) \frac{1}{2}$, $E\left[T_{i} \mid X^{\eta}=x\right]=\eta_{T}(\eta) x+\left(1-\eta_{T}(\eta)\right) \frac{1}{2}$, and $\mu(x, \eta)=\eta_{Q}(\eta) \frac{x}{2}$.

We define our two second stage games and their corresponding equilibrium concepts in the next section. An equilibrium of the first stage is defined as an information precision $\eta_{*} \in[0,1]$ such that $\eta_{*}$ is a symmetric Nash equilibrium to the game defined by the equilibrium payoffs of the second stage game. The analysis of asymmetric equilibria is limited by the complexity of the continuation games. The larger the number of bidders with different information precision, the more difficult is the characterization of their equilibria. ${ }^{6}$

\section{Analysis of the auction games}

In this section, we study the second stage. Since we are interested in symmetric equilibria of the first stage, we restrict our study to the case in which only one bidder chooses a different information precision. We call this bidder the deviating bidder and denote her index as $d \in I$ and her information precision as $\eta_{d}$. We refer to the other bidders as non-deviating bidders and to their information precision as $\eta$.

5 A sufficient condition for this regularity assumption is that the cumulative distribution function of the conditional expected value $E\left[Q_{i} \mid X_{i}^{\eta}\right]$, is log-concave. This assumption is satisfied by many distribution functions, see Bagnoli and Bergstrom (2005). For instance any distribution function of the form $F(q)=q^{r}, r \geqslant 1$, and any truncated exponential, normal, logistic, extreme-value, chi-square, chi, and Laplace distributions.

6 In any case, the existence of asymmetric equilibrium would depend at the end on the assumptions on the concavity of the cost function. 


\subsection{The open auction}

In this section, we assume that in the second stage bidders play the English auction studied by Krishna (2003). In fact, we shall adapt his analysis, in particular his Lemma 1, to our framework to construct an ex post equilibrium that implements the ex post efficient allocation. ${ }^{7}$ We refer the reader to Krishna's paper for the details on the definition of the game, strategy space and equilibrium concept.

To construct the equilibrium strategies, we first introduce an auxiliary function $\phi_{O}:[x, \bar{x}] \subset$ $[0,1] \rightarrow[0,1]$ defined implicitly for a given vector $x_{-i, d} \in[0,1]^{n-2}$ by:

$$
\begin{aligned}
& E\left[T_{d}+\sum_{j=1}^{n} Q_{j} \mid X_{d}^{\eta_{d}}=x, X_{i}^{\eta}=\phi_{O}(x), X_{-i, d}^{\eta}=x_{-i, d}\right] \\
& \quad=E\left[T_{i}+\sum_{j=1}^{n} Q_{j} \mid X_{d}^{\eta_{d}}=x, X_{i}^{\eta}=\phi_{O}(x), X_{-i, d}^{\eta}=x_{-i, d}\right],
\end{aligned}
$$

where $X_{-i, d}^{\eta}$ denotes the vector of all the signals of the non-deviating bidders but $i$, and $[\underline{x}, \bar{x}]$ the set of $x$ 's for which the equation has a solution. Before we provide an intuitive explanation of this equation note it can be simplified after some trivial algebra to the following equation,

$$
E\left[T_{d} \mid X_{d}^{\eta_{d}}=x\right]=E\left[T_{i} \mid X_{i}^{\eta}=\phi_{O}(x)\right],
$$

and hence, $\phi_{O}$ is independent of the particular vector $x_{-i, d}$ chosen.

Our assumptions of differentiability and monotonicity of $E\left[T_{i} \mid X_{i}^{\eta_{i}}=x\right]$ imply that the function $\phi_{O}$ is strictly increasing and has a continuous differential bounded from above and bounded away from zero; the inverse function exists and we denote it by $\phi_{O}^{-1}$; and the graph of $\phi_{O}$ starts at a point in either the lower bound or the left bound of $[0,1]^{2}$ and finishes at either the upper bound or the right bound of $[0,1]^{2}$. However, our assumptions do not guarantee that $\phi_{O}$ and $\phi_{O}^{-1}$ have domain $[0,1]$. We find it convenient to define the extension of $\phi_{O}$ to the domain $[0,1]$ as follows,

$$
\hat{\phi}_{O}\left(x_{d}\right) \equiv \begin{cases}0 & \text { if } x_{d} \in[0, x), \\ \phi_{O}\left(x_{d}\right) & \text { if } x_{d} \in[\underline{x}, \bar{x}], \\ 1 & \text { if } x_{d} \in(\bar{x}, 1],\end{cases}
$$

and a similar expression for the extension of $\phi_{O}^{-1}$ that we denote $\hat{\phi}_{O}^{-1}$. Note that we are abusing of the notation since $\hat{\phi}_{O}^{-1}$ is the inverse function of $\hat{\phi}_{O}$ only in the range in which $\hat{\phi}_{O}$ is invertible, this is when $\hat{\phi}_{O}(x)=\phi_{O}(x)$. In what follows, we shall call the extension of a continuous increasing function $\phi: A \subset[0,1] \rightarrow[0,1]$, for $A$ a closed interval, to a function constructed as above. We shall also use $\hat{\phi}^{-1}$ to denote the extension of the inverse.

Intuitively, the function $\phi_{O}$ gives us the type of a non-deviating bidder with the same conditional expected value as a given type of a deviating bidder. We have seen above that this is independent of the vector of other bidder's types in which we condition. Note that since an increase in a bidder's type increases her conditional expected value more than the conditional expected value of the other bidders, $\phi_{O}$ characterizes the efficient allocation. This is, it is ex post

7 Note that this is possible because our assumption that the bidders' values are additively separable implies trivially Krishna's average and cyclical single crossing conditions. 
efficient to give the good to the deviating bidder if the realization of $\left(X_{d}^{\eta_{d}}, \max _{i \neq d}\left\{X_{i}^{\eta}\right\}\right)$ is to the right and down of the graph of $\phi_{O}$, otherwise it is ex post efficient to give it to the non-deviating bidder with highest type.

A strategy must specify whether the bidder remains active at a given price as a function of her private type, and the vector of prices and identities of the bidders that have already dropped out, if any. We characterize the strategy of Bidder $i$ with a bid function $b_{i}\left(x \mid\left\{\left(p_{l}, j_{l}\right)\right\}_{l=1}^{k}\right)$ that specifies the price at which the bidder quits if $k$ bidders with identities $j_{1}, j_{2}, \ldots, j_{k}$ already dropped out at the respective prices $p_{1} \leqslant p_{2} \leqslant \cdots \leqslant p_{k}$. In what follows, and in order to simplify the notation, we adopt the convention that for any $y_{r}$, the expression $\left\{y_{r}\right\}_{r=1}^{0}$ denotes the empty set. We also let $\Sigma$ denote $\left\{b_{j_{l}}^{O}\left[X_{j_{l}}^{\eta_{j_{l}}} \mid\left\{\left(p_{q}, j_{q}\right)\right\}_{q=1}^{l-1}\right]=p_{l}\right\}_{l=1}^{k}$ where $\eta_{j_{l}}=\eta$ for any $j_{l} \neq d$. Our proposed strategy is defined recursively starting at $k=0$ as follows:

- Bid function of the deviating bidder:

$$
b_{d}^{O}\left[x_{d} \mid\left\{\left(p_{l}, j_{l}\right)\right\}_{l=1}^{k}\right] \equiv E\left[T_{d}+\sum_{j=1}^{n} Q_{j} \mid X_{d}^{\eta_{d}}=x_{d},\left\{X_{j}^{\eta}=\hat{\phi}_{O}\left(x_{d}\right)\right\}_{\substack{j \notin\left\{j_{l}\right\}_{l=1}^{k} \\ j \neq d}}, \Sigma\right] .
$$

- Bid function of a non-deviating bidder $i$, if the deviating bidder has not quit yet:

$$
b_{i}^{O}\left[x_{i} \mid\left\{\left(p_{l}, j_{l}\right)\right\}_{l=1}^{k}\right] \equiv E\left[T_{i}+\sum_{j=1}^{n} Q_{j} \mid X_{d}^{\eta_{d}}=\hat{\phi}_{O}^{-1}\left(x_{i}\right),\left\{X_{j}^{\eta}=x_{i}\right\}_{\substack{j \notin\left\{j_{l} l_{l=1}^{k} \\ j \neq d\right.}}, \Sigma\right] .
$$

- Bid function of a non-deviating bidder $i$, if the deviating bidder has already quit:

$$
b_{j}^{O}\left[x_{i} \mid\left\{\left(p_{l}, j_{l}\right)\right\}_{l=1}^{k}\right] \equiv E\left[T_{i}+\sum_{j=1}^{n} Q_{j} \mid\left\{X_{j}^{\eta}=x_{i}\right\}_{j \notin\left\{j_{l}\right\}_{l=1}^{k}}, \Sigma\right] .
$$

The definition of $\phi_{O}$ in Eq. (1) implies that if $x_{i}=\phi_{O}\left(x_{d}\right)$, and thus $\phi_{O}^{-1}\left(x_{i}\right)=x_{d}$, then $b_{d}^{O}\left(x_{d} \mid\left\{\left(p_{l}, j_{l}\right)\right\}\right)=b^{O}\left(x_{i} \mid\left\{\left(p_{l}, j_{l}\right)\right\}\right)$, see Eqs. (1), (3) and (4). This means that a bidder bids her expected value conditional on her private information, the information that she can infer from the prices at which other bidders have already quit, and the information that can be inferred from the hypothetical event that all the remaining bidders in the auction quit at the same price as her. Moreover, the monotonicity of the bid functions and the fact that all deviating bidders use the same bid function implies that the efficient allocation is implemented when bidders use our proposed bid functions.

Proposition 1. The bid function $b_{d}^{O}$ for the deviating bidder and the bid functions $b^{O}$ for all the non-deviating bidders is an equilibrium point of the open auction.

Note, however, that there may be other equilibria. This is not surprising since open auctions have typically multiple equilibria. ${ }^{8}$ We find our equilibrium to be focal since it is ex post efficient. Note also that although there may be other ex post efficient equilibria, they imply the same results in Sections 4 and 5. This is basically because they share that the deviating bidder wins if and only

8 This was well known since Milgrom and Weber (1982a). Bikhchandani and Riley (1991) provides a careful analysis of the multiplicity of equilibria under the pure common value assumption and Bikhchandani et al. (2002) for symmetric equilibria in strictly increasing strategies in a model with ex ante symmetric bidders and general interdependent values. 
if her type is greater than the image of the maximum type of the non-deviating bidders through $\phi_{O}$ which determines the expected utility of a deviating bidder up to type zero by Lemma 3 . Besides, the utility of a type zero does not upset our results. It is equal to zero under the common value assumption for any efficient equilibrium because $\phi_{O}(0)=0$ by Lemma 4 . Moreover, it does not upset the results under the private value assumption because they require a large $n$ and in this case, the expected utility of a type zero tends to zero in any case.

\subsection{The sealed bid auction}

In this section, we assume that in the second stage bidders play the sealed bid auction introduced by Vickrey (1961) and called second price auction. We refer the reader to Milgrom and Weber's (1982a) paper for the description of the mechanism, strategies and equilibrium concept.

We follow similar steps as in the previous section: we define an auxiliary function that we use to construct the equilibrium strategies. Let $\Gamma$ be the set of continuous and strictly increasing functions $\phi: A \subset[0,1] \rightarrow[0,1]$ that satisfy the equation below and with a graph that starts at the lower or left bound of $[0,1]^{2}$ and ends at its upper or right bound.

$$
\begin{aligned}
& E\left[T_{d}+\sum_{j=1}^{n} Q_{j} \mid X_{d}^{\eta_{d}}=x, \max \left\{X_{j}^{\eta}\right\}_{j \neq d}=\phi(x)\right] \\
& \quad=E\left[T_{i}+\sum_{j=1}^{n} Q_{j} \mid X_{i}^{\eta}=\max \left\{\left\{X_{j}^{\eta}\right\}_{j \neq d, i} \cup\left\{\phi\left(X_{d}^{\eta_{d}}\right)\right\}\right\}=\phi(x)\right] .
\end{aligned}
$$

Note that for any $\phi \in \Gamma$ we can define its extension, say $\hat{\phi}$, and hence the following bid functions:

- Bid function of the deviating bidder:

$$
b_{d}^{S}\left(x_{d}\right) \equiv E\left[T_{d}+\sum_{j=1}^{n} Q_{j} \mid X_{d}^{\eta_{d}}=x_{d}, \max \left\{X_{j}^{\eta}\right\}_{j \neq d}=\hat{\phi}\left(x_{d}\right)\right] .
$$

- Bid function of a non-deviating bidder $i \neq d$ :

$$
b^{S}\left(x_{i}\right) \equiv E\left[T_{i}+\sum_{j=1}^{n} Q_{j} \mid X_{i}^{\eta}=\max \left\{\left\{X_{j}^{\eta}\right\}_{j \neq d, i} \cup\left\{\hat{\phi}\left(X_{d}^{\eta_{d}}\right)\right\}\right\}=x_{i}\right] \text {, }
$$

where to get a compact notation we have used the convention that for $x_{i}<\hat{\phi}(0)$,

$$
\left\{\max \left\{\left\{X_{j}^{\eta}\right\}_{j \neq d, i} \cup\left\{\hat{\phi}\left(X_{d}^{\eta_{d}}\right)\right\}\right\}=x_{i}\right\} \equiv\left\{\left\{X_{j}^{\eta} \leqslant x_{i}\right\}_{j \neq d, i} \cup\left\{X_{d}^{\eta_{d}}=0\right\}\right\} .
$$

Note that by Eq. (6) these bid functions are such that if $x_{i}=\phi\left(x_{d}\right)$, then $b^{S}\left(x_{i}\right)=b_{d}^{S}\left(x_{d}\right)$. Thus, a bidder bids her expected value conditional on her private information and the information inferred from the hypothetical event that she is tying with the highest bid of the other bidders. We can also deduce that a deviating bidder with type $x_{d}$ bids higher (lower) than a non-deviating bidder with type $x_{i}$ for any $\left(x_{d}, x_{i}\right) \in[0,1]^{2}$ to the right and down (left and up) of the graph of $\phi$.

In general, it is not easy to show that $\Gamma$ is non-empty. It requires proving the existence of a solution to a differential equation with boundary conditions at singular points. For the same reasons, it is also complex to characterize its properties. We deal with these issues in Appendix A.2, 
in particular in the proof of Proposition 2. Here, we introduce a function that let us follow the analysis more easily. Let $\phi_{*}:\left[\underline{x}^{\prime \prime}, \bar{x}^{\prime \prime}\right] \subset[0,1] \rightarrow[0,1]$ be defined implicitly by:

$$
\begin{aligned}
& E\left[T_{d}+\sum_{j=1}^{n} Q_{j} \mid X_{d}^{\eta_{d}}=x, \max \left\{X_{j}^{\eta}\right\}_{j \neq d}=\phi_{*}(x)\right] \\
& \quad=E\left[T_{i}+\sum_{j=1}^{n} Q_{j} \mid X_{i}^{\eta}=\max \left\{X_{j}^{\eta}\right\}_{j \neq d, i}=\phi_{*}(x), X_{d}^{\eta_{d}} \leqslant x\right],
\end{aligned}
$$

where $\left[\underline{x}^{\prime \prime}, \bar{x}^{\prime \prime}\right]$ is the set of $x$ 's for which the equation has a solution. This equation can be simplified to:

$$
E\left[T_{d} \mid X_{d}^{\eta_{d}}=x\right]-E\left[T_{i} \mid X_{i}^{\eta}=\phi_{*}(x)\right]+\mu\left(x, \eta_{d}\right)-\mu\left(\phi_{*}(x), \eta\right)=0 .
$$

Our assumptions of differentiability and monotonicity of $E\left[T_{i} \mid X_{i}^{\eta}=x\right]$ and $\mu(x, \eta)$ imply that the function $\phi_{*}$ is strictly increasing and has a differential bounded from above and away from zero, and the graph of $\phi_{*}$ starts at a point in either the lower bound or the left bound of $[0,1]^{2}$ and finishes at either the upper bound or the right bound of $[0,1]^{2}$.

Proposition 2. For any $n,{ }^{9}$ there exists a function in $\Gamma$ that we denote by $\phi_{S, n}$ such that the corresponding bid functions in Eqs. (7) and (8) characterize an equilibrium point of the sealed bid auction.

The function $\phi_{S, n}$ has the following properties:

1. For any $x \in[0,1], \hat{\phi}_{S, n}(x)$ is between $\hat{\phi}_{O}(x)$ and $\hat{\phi}_{*}(x)$, strictly if $\left(x, \hat{\phi}_{S, n}(x)\right) \in(0,1)^{2}$ and $\hat{\phi}_{O}(x) \neq \hat{\phi}_{*}(x)$.

2. If $\hat{\phi}_{O}^{-1}(1)<\hat{\phi}_{*}^{-1}(1)$, then $\hat{\phi}_{O}^{-1}(1)<\hat{\phi}_{S, n}^{-1}(1)$; and if $\hat{\phi}_{O}(1)<\hat{\phi}_{*}(1)$, then $\hat{\phi}_{O}(1)<\hat{\phi}_{S, n}(1)$.

3. For any $x \in[0,1], \hat{\phi}_{S, n+1}(x)$ is between $\hat{\phi}_{S, n}(x)$ and $\hat{\phi}_{*}(x)$.

To interpret the properties of $\phi_{S, n}$ note the similarities between Eq. (6) and the equations that define $\phi_{O}$ and $\phi_{*}$, Eq. (1) and Eq. (9) respectively. The differences appear in the right-hand side. In the three cases, it equals the expected value of a non-deviating bidder conditional on the maximum of the other bidders' types (the deviating bidder's type rescaled by the corresponding function $\phi$ ) equal to a certain number, ${ }^{10}$ but differ in who has the maximum type: the deviating bidder in Eq. (1), a non-deviating bidder in Eq. (9), and in Eq. (6) the intermediate case in which it can be either the deviating bidder or another of the non-deviating bidders. This explains the items 1 and 2 of the proposition above. Besides, the item 3 corresponds to the intuition that a greater number of bidders should increase the probability that the maximum type is the type of a non-deviating bidder.

Note that we do not expect the equilibrium to be unique in general. Actually, there are three potential sources of multiplicity even when we restrict to equilibria in strictly increasing bid functions and symmetric in the sense that all the non-deviating bidders use the same bid function.

\footnotetext{
9 The solutions in $\Gamma$ typically depend on $n$. See the proof of the proposition.

10 Note that the right-hand side of Eq. (1) does not condition on the event that the maximum of type the other nondeviating bidders is less than the corresponding threshold as required. Instead it conditions on an arbitrary vector of types $x_{-i, j}$. However, since Eq. (1) defines the same function independently of the vector $x_{-i, j}$ chosen, we can obtain the required equation simply by integrating with respect to all vectors $x_{-i, j}$ below the appropriate threshold.
} 
To understand the first source recall that by a well-known equilibrium condition in the analysis of second price auctions, a bidder must be indifferent between winning and losing at the price that she bids if her bid lies in the support of the maximum of the other bidders' bids. This condition applied to our model implies that if both a deviating bidder with type $x$ and a non-deviating bidder with type $\phi(x)$ bid $p$, then the left- and right-hand sides of Eq. (6) must be equal to $p$. Thus it implies our equation (1) and our bid functions for the types that bid in the intersection of the range of both bid functions. Consequently, our equilibrium is the equilibrium that satisfies this condition for the largest possible set of types, i.e. it is the equilibrium in which the intersection between the range of the bid function of the deviating bidder and the bid function of the non-deviating bidders is maximum. There might be other equilibria in which this intersection is smaller, but they require the bid functions to be discontinuous ${ }^{11}$ at some arbitrary points and thus it seems more difficult that bidders can coordinate on them.

For the second source, note that in our equilibrium there may be types of a bidder bidding outside the support of the maximum of the other bidders' bids. For instance, this is the case for types of the deviating bidder below $\phi_{S, n}^{-1}(0)$ (when it is defined and not equal to zero). In this case, the bidder is indifferent between any bid below $b^{S}(0)$ because they all lose with probability one. Thus, it is not surprising that we can find other equilibria that only differ in the bids of these types. This is also the case for types of the non-deviating bidder less than $\phi_{S, n}(0)$ and for a symmetric reason for types of the deviating bidder greater than $\phi_{S, n}^{-1}(1)$. In the last case because several bids let her win with probability one and pay the same price. However, note that the last case does not affect the equilibrium outcome because any of these bids induce the same winner, the deviating bidder, and price, the highest bid of the non-deviating bidders. Note also that the other two cases do not arise under the Q-information assumption because ${ }^{12} \phi_{S, n}(0)=0$ and $\phi_{S, n}^{-1}(0)=0$. They, however, might give rise to multiplicity of equilibria under the T-information assumption. But, this does not upset our results because they require a large $n$, and as $n$ tends to infinity the effect of the bids of low types becomes negligible.

Finally, the last source of multiplicity is that Eq. (6) boils down to a differential equation, see Lemma 10 in Appendix A.2, that in principle may have multiple solutions. ${ }^{13}$ Here, our approach is to choose the solution that implements the most efficient allocation, see the proof of Proposition 2 and in particular item 2 of Lemma 13. This selection argument is consistent with the one used for the open auction.

\subsection{Strategic effects of information acquisition}

In this subsection, we characterize some properties of the equilibrium bid functions that we use later to prove that a deviating bidder has different incentives to acquire information in each auction. We start with an intuitive discussion on how the information acquisition of the deviating bidder changes the equilibrium bids of non-deviating bidders. Throughout this discussion, we

11 Alternatively, the whole range of the bid function of the deviating bidder might be either above or below the range of the bid function of the non-deviating bidders. But it may be shown that these other equilibria are in weakly dominated strategies.

12 To see why note that under this assumption it is easy to derive from Eq. (2) that $\phi_{O}(0)=0$ and from Eq. (10) that $\phi_{*}(0)=0$, and thus by the first item of Proposition $2 \phi_{S, n}(0)=0$ and $\phi_{S, n}^{-1}(0)=0$.

13 Technically, there could be multiple solutions to the associated differential equation, Eq. (13) in Appendix A.2, that satisfy the conditions of Proposition 2 because they imply boundary conditions at singular points. Thus, uniqueness cannot be established by standard theorems. 
concentrate on the case in which the deviating bidder wins the auction; otherwise she gets zero expected utility in any case.

We first consider the Q-information assumption, i.e. information acquisition about the common value. As Eqs. (4) and (8) show, the effect on bids in both auctions happens through the hypothetical event that the bidder submits the same bid as the highest bid of the rivals. This hypothetical event is the intersection of two others: that the bidder's bid is a lower bound for the highest bid of the other bidders, the loser's curse, and that it is an upper bound, the winner's curse. $^{14}$

We may expect information acquisition by the deviating bidder to have stronger effects on the loser's curse of the non-deviating bidders in the open auction than in the sealed bid auction. To see why, note that in the case we are interested in, a non-deviating bidder can determine that the deviating bidder has the highest bid in the open auction but not in the sealed bid auction. Thus, while the loser's curse determines a lower bound to the type of the deviating bidder in the open auction, it only implies that this may be the case with some probability in the sealed bid auction. This can be seen comparing Eqs. (4) and (8).

On the contrary, we may expect similar effects of information acquisition in the winner's curse in both auction formats. The reason is that the winner's curse fixes an upper bound on the type of the deviating bidder in either case: in the open auction because the bidder can determine that the deviating bidder has the highest bid of the other bidders, and in the sealed bid auction because it implies an upper bound on the bids of all the other bidders.

The loser's curse means good news about the common value which information acquisition converts into better news, and thus induces higher bidding. Since this effect must be stronger in the open auction than in the sealed bid auction, we expect the deviating bidder to face relatively fiercer competition, and thus to win relatively less often in the open auction than in the sealed bid auction. The next lemma formalizes this conjecture.

\section{Lemma 1. Under the Q-information assumption:}

1. If $\eta_{d}>\eta$, then $\hat{\phi}_{S, n}(x)>\hat{\phi}_{O}(x)=x$, for any $x \in(0,1)$.

2. If $\eta_{d}<\eta$, then $\hat{\phi}_{S, n}(x)<\hat{\phi}_{O}(x)=x$, for any $x \in(0,1)$.

Consider, next, the T-information assumption, i.e. information acquisition about the private value. In this case, the effect on bids of non-deviating bidders is indirect. Better information induces more disperse bidding, and thus that the deviating bidder submits her high bids with lower types. ${ }^{15}$ As Fig. 1 illustrates, this shift in the bid function makes the bad news of the winner's curse worse and the good news of the loser's curse not so good.

Both effects induce lower bidding by non-deviating bidders, but we expect the reduction to be greater in the open auction than in the sealed bid auction because of the difference in the loser's curse we explained above. Consequently, the high bids of non-deviating bidders should decrease and relatively more in the open auction than in the sealed bid auction. Thus, a deviating bidder bidding high, i.e. with a high type, should outbid non-deviating bidders more often in the open auction than in the sealed bid auction. The next lemma proves this conjecture.

14 The formal definition of loser's curse and winner's curse as statistical events was introduced by Pesendorfer and Swinkels (1997).

15 Similarly, the low bids are submitted by higher types, though this effect does not play any role in our analysis. 


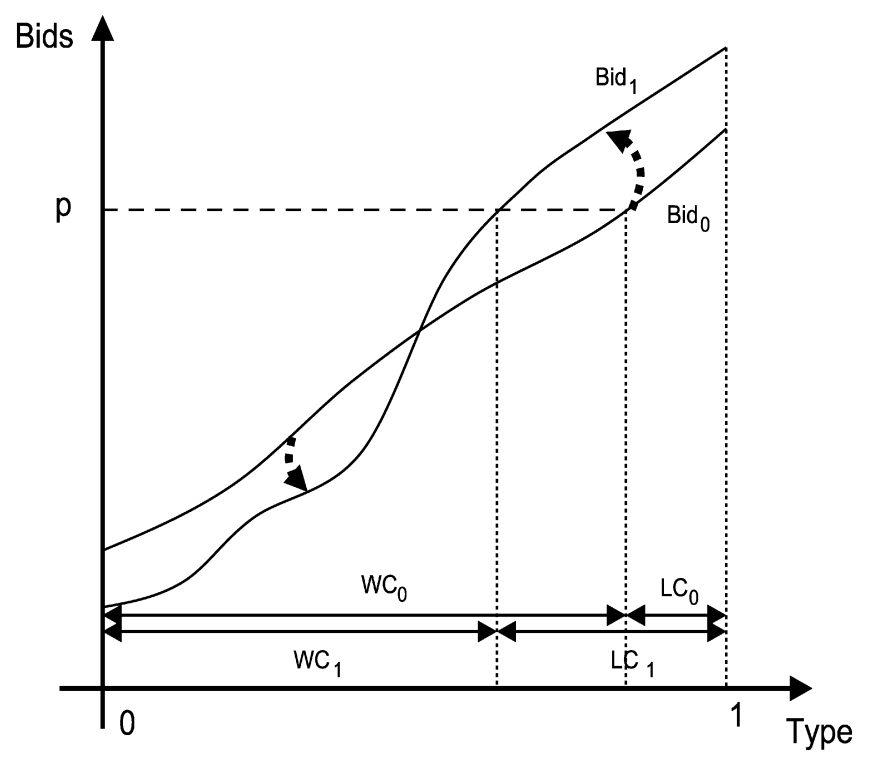

Fig. 1. Change in the winner's curse $\left(W C_{0} \rightarrow W C_{1}\right)$ and loser's curse $\left(L C_{0} \rightarrow L C_{1}\right)$ for a high bid $p$ as a consequence of a change in the bid function $\left(\operatorname{Bid}_{0} \rightarrow \operatorname{Bid}_{1}\right)$.

Lemma 2. Under the T-information assumption:

1. For $\eta_{d}>\eta$, there exists an $\epsilon>0$ such that $\hat{\phi}_{O}^{-1}(x)<\hat{\phi}_{S, n}^{-1}(x) \leqslant \hat{\phi}_{*}^{-1}(x)<x$ for $x \in$ $(1-\epsilon, 1]$.

2. For $\eta_{d}<\eta$, there exists an $\epsilon>0$ such that $\hat{\phi}_{O}(x)<\hat{\phi}_{S, n}(x) \leqslant \hat{\phi}_{*}(x)<x$ for $x \in(1-\epsilon, 1]$.

\section{Analysis of the game of information acquisition}

In this section, we study the first stage game, the game of information acquisition, assuming the bidders' continuation payoffs are given by the equilibrium in Propositions 1 and 2. To compute these payoffs, note that under our assumption of independent types, and by the arguments of the analysis of Myerson (1981), the allocation function and the expected utility of the minimum types characterize the expected utility of the bidders. Moreover, the additive separability of the bidders' utility function makes specially simple the expression of the bidder's expected utility as the next lemma shows.

Lemma 3. Suppose Bidder $d \in I$ wins if and only if $\phi\left(X_{d}^{\eta_{d}}\right) \geqslant \max \left\{X_{i}^{\eta_{i}}\right\}_{i \neq d}$ in the equilibrium of an auction. Then, her ex ante expected utility is equal to:

$$
\int_{0}^{1}(1-x) \phi(x)^{n-1} \frac{\partial E\left[T_{d}+Q_{d} \mid X_{d}^{\eta_{d}}=x\right]}{\partial x} \mathrm{~d} x
$$

plus the expected utility that Bidder d gets when she has type 0. 
We denote by $U^{a}\left(\eta_{d}, \eta\right)$ the expected utility of a deviating bidder in auction format $a \in\{S, O\}$ when her information precision is $\eta_{d}$ and all the other bidders' information precision is equal to $\eta$. We also let $\frac{\Delta U^{a}}{\Delta \eta}\left(\eta_{d}, \eta\right) \equiv \frac{U^{a}\left(\eta_{d}, \eta\right)-U^{a}(\eta, \eta)}{\eta_{d}-\eta}$.

Lemmas 1, 2 and 3 imply the following result:

Proposition 3. For any $\eta_{d} \neq \eta$,

1. $\frac{\Delta U^{S}}{\Delta \eta}\left(\eta_{d}, \eta\right)>\frac{\Delta U^{O}}{\Delta \eta}\left(\eta_{d}, \eta\right)>0$ under the $Q$-information assumption.

2. $\frac{\Delta U^{O}}{\Delta \eta}\left(\eta_{d}, \eta\right)>\frac{\Delta U^{S}}{\Delta \eta}\left(\eta_{d}, \eta\right)>0$ under the T-information assumption if $n$ is large enough.

That the value of additional private information about the common value is positive contrasts with the results of Hernando-Veciana and Tröge (2005) which show that the opposite may happen. To understand this difference, note that our analysis has shown that information acquisition affects the other bidders' bids through the change in the informativeness of one's bid. In this respect, our two models differ in that information acquisition about the common value in Hernando-Veciana and Tröge's model makes the bidder's bid less informative of the private value whereas under our assumptions it remains the same, see also our Conclusions.

Finally, we use Proposition 3 to derive a comparison of the equilibrium level of information acquisition. In order to ensure existence and uniqueness of the equilibrium, we need that the functions $U^{a}\left(\eta_{d}, \eta\right), a \in\{O, S\}$, satisfy some regularity conditions, in particular:

[A.1] The functions $U^{a}\left(\eta_{d}, \eta\right)$ are continuous.

[A.2] The limits $\lim _{\eta_{d} \rightarrow \eta} \frac{\Delta U^{a}}{\Delta \eta}\left(\eta_{d}, \eta\right)$ exist and are continuous in $\eta$.

[A.3] The properties of Proposition 3 are also satisfied in the $\operatorname{limit}_{\eta_{d} \rightarrow \eta} \frac{\Delta U^{a}}{\Delta \eta}\left(\eta_{d}, \eta\right)$.

[A.4] The bound on $n$ in the second item of Proposition 3 and the extension implied by [A.3] holds uniformly for any $\left(\eta_{d}, \eta\right) \in[0,1]^{2}$.

Proposition 4. Suppose ${ }^{16} C(\eta)=\frac{\alpha}{2} \eta^{2}$, for $\alpha \in(0, \infty)$. If the functions $U^{a}, a \in\{S, O\}$, satisfy conditions [A.1]-[A.4], there exists a unique equilibrium information precision in the sealed bid $\eta_{*}^{S} \in[0,1]$, and in the open auction $\eta_{*}^{O} \in[0,1]$, if $\alpha$ is sufficiently large. Moreover,

1. $\eta_{*}^{S}>\eta_{*}^{O}$ under the $Q$-information assumption.

2. $\eta_{*}^{S}<\eta_{*}^{O}$ under the T-information assumption, if $n$ is large enough.

A careful reader may worry that our conditions [A.1]-[A.4] are defined on derived objects, the functions $U^{a}\left(\eta_{d}, \eta\right)$, rather than on the primitives of the model. Note that we can show that [A.1] and [A.2] hold true for $a=O$ from Lemma 3, the application of the implicit function theorem to Eq. (2) and our assumptions on $E\left[V_{i} \mid T_{i}^{\eta}=x\right]$. Regretfully, we cannot do a similar argument for $U^{S}\left(\eta_{d}, \eta\right)$ since $\phi_{S, n}$ is the solution to a differential equation with boundary conditions at singular points and hence no standard theorem guarantees its continuity and differentiability

16 Our assumptions on the cost function are an adaptation of those made by Persico (2000). They ensure two things, that the set of $\eta_{d}$ 's that maximize $U^{a}\left(\eta_{d}, \eta\right)-C\left(\eta_{d}\right)$, i.e. the best response, is single-valued and that $\lim _{\eta_{d} \rightarrow \eta} \frac{\Delta U^{a}}{\Delta \eta}\left(\eta_{d}, \eta\right)-$ $C^{\prime}(\eta)$ decreases in $\eta$. Both conditions are used to guaranty existence and uniqueness, respectively, of the equilibrium level of information acquisition. 
with respect to $\left(\eta_{d}, \eta\right)$. We provide in Appendix A.3 an example that illustrates that there exists functions $\phi_{S, n}$ that satisfy the properties required for [A.1] and [A.2]. We also show that the same example verifies [A.3] and [A.4].

\section{Revenue and efficiency}

Different levels of information acquisition imply different levels of efficiency and auctioneer's expected revenue. We can thus compare our two auction formats in these two dimensions assuming that bidders play the equilibria in Proposition 4.

Suppose that the auctioneer does not put any value on keeping the object; then the social surplus generated in the auction excluding information acquisition costs is simply the expected value of the bidder who gets the object. In this sense, we say that an auction is more allocatively efficient ${ }^{17}$ than another one if the expected value of the winning bidder in the former auction is larger than in the latter one. We also say that an auction is more ex ante efficient than another auction if the difference between the expected value of the winning bidder and the sum of all the information acquisition costs is larger in the former auction than in the latter one.

We first consider a set of auction models that include our two auctions when all bidders choose the same level of information precision. We call a standard-symmetric auction, an auction model together with an equilibrium in which all bidders observe signals of the same information precision $\eta$, the bidder with highest type gets the object, and only the winning bidder makes a payment to the auctioneer.

Two standard-symmetric auctions with the same $\eta$ induce the same allocation and thus achieve the same level of allocative efficiency and ex ante efficiency. Moreover:

Lemma 4. In a standard-symmetric auction with bidders' information precision $\eta$ :

1. The allocative efficiency is increasing in $\eta$ under the T-information assumption ${ }^{18}$ and remains constant under the $Q$-information assumption.

2. The ex ante efficiency is decreasing in $\eta$ under the $Q$-information assumption.

Intuitively, bidders' values only differ in their private component. Hence, an increase in the bidders' information precision can increase the allocative efficiency under the T-information assumption but not under the Q-information assumption. Moreover, ex ante efficiency decreases in the latter case since information is costly.

The combination of Lemma 4 with Proposition 4 implies the following.

Corollary 1. Under the assumptions of Proposition 4:

1. The open auction is more allocatively efficient than the sealed bid auction under the Tinformation assumption if $n$ is large enough.

2. The open auction is more ex ante efficient than the sealed bid auction under the $Q$ information assumption.

17 Ex post efficiency is a reduced form for other objectives. This is the case, for instance, of a social planner maximizing the consumer surplus who auctions a monopoly license among firms that differ in their (constant) marginal costs.

18 Ganuza and Penalva-Zuasti (2006) prove a similar result in a pure private value model. They also provide a result with respect to expected revenue similar to the second item of Lemma 5. 
Similarly, two standard-symmetric auctions with the same level of information precision induce the same expected revenue to the auctioneer by a version of the revenue equivalence theorem. Moreover:

Lemma 5. In a standard-symmetric auction with bidders' information precision $\eta$ :

\section{The expected revenue of the auctioneer is decreasing in $\eta$ under the $Q$-information assump- tion.}

2. The expected revenue of the auctioneer is increasing in $\eta$ under the T-information assumption if $n$ is large enough.

Leaving aside information acquisition costs, the auctioneer's expected revenue is equal to the difference between the expected social surplus generated in the auction and the expected utility of all the bidders. It is easy to deduce from Lemma 3 that the bidders' expected utility is increasing in $\eta$. This is actually a consequence of our assumption of independence of the bidders' private information. The intuitive reason is that bidders' informational rents increase. ${ }^{19}$ As a consequence, and because of Lemma 4, the expected revenue decreases with $\eta$ under the Q-information assumption. Under the T-information assumption the situation is different because the expected social surplus increases with $\eta$, see Lemma 4 . Thus, the auctioneer's expected revenue increases if the sum of bidders' expected utilities is negligible. This is the case when $n$ is large enough.

We can deduce from Lemma 5 and Proposition 4 the following.

Corollary 2. Under the assumptions of Proposition 4, the open auction gives greater expected revenue to the auctioneer than the sealed bid auction: under the Q-information assumption, for any $n$; and under the T-information assumption, if $n$ is large enough.

\section{Conclusions}

In this paper, we have studied a theoretical model of observable information acquisition which distinguishes between information acquisition about the common value and information acquisition about the private value. Our particular assumptions on this distinction imply that the bid of a bidder who acquires more information about, for instance, the common value becomes more informative about the common value while it remains equally informative with respect to the private value. This distinction has allowed us to provide clear-cut rankings of our two auctions with respect to the incentives to acquire information.

Note, however, that our model seems to lie somewhere between two existing branches of the literature of information acquisition in auctions. First, the models of Bergemann and Välimäki (2002) and Hagedorn (2004), in which information acquisition makes the bidder's action more informative with respect to both the common and the bidder's private value simultaneously. Second, the models of Larson (2004) and Hernando-Veciana and Tröge (2005) in which information

19 This is not necessarily true if bidders' private information is affiliated. For instance, in the mineral rights model of Milgrom and Weber (1982a) in which each bidder observes a noisy signal of the common value their expected utility goes to zero as their signal becomes perfectly informative. Note that this also implies that the auctioneer's expected revenue may increase with the amount of bidders' private information about the common value. Independence, however, may be a good approximation if affiliation is weak, as it may happen, for instance, when there is much uncertainty. Moreover, there are real-life examples in which this assumption is reasonable even with common values, see Bergemann and Välimäki (2002). 
acquisition makes the bidder's bid more informative with respect to the common value but in general $^{20}$ less informative with respect to the bidder's private value.

Our results provide a conjecture for the former alternative models. This is because information acquisition affects the other bidders' bids through changes in the informativeness of the bidders' bid with respect to the private and common value. Thus, we expect, for instance, that the sealed bid auction gives greater incentives to acquire information than the open auction in the model of Hernando-Veciana and Tröge (2005). The reason is that in their model information acquisition increases the informativeness of the bidder's bid with respect to the common value but decreases it with respect to the private value. Our results suggest that both effects change the other bidders' bids such that the incentives to acquire information are greater in the sealed bid auction than in the open auction.

\section{Acknowledgments}

I thank seminar participants at ELSE (University College London), University Carlos III, University of Barcelona, University of Alicante, Tilburg University, Queen's University Belfast, WZB (Berlin) and Université Paris I (Sorbonne), and specially the associate editor, Marco Celentani, Natalia Fabra, Maria-Ángeles de Frutos, Francisco Marhuenda, Juan Pablo Rincón, Francesco Squintani and Luis Úbeda. The usual disclaimer applies. I am also grateful for the financial support of the European Commission through a Marie Curie scholarship, the Spanish Ministerio de Educación y Ciencia (project SEJ2006-11665-C02-02) and of the Instituto Valenciano de Investigaciones Económicas.

\section{Appendix A}

This appendix has three parts. In Appendix A.1 we show in what sense our assumptions Q-information and T-information correspond to more informative signals. In Appendix A.2 we include the proofs of all the lemmas and propositions in the main text. Appendix A.3 illustrates assumptions [A.1]-[A.4] with Example A.

\section{A.1. More informative signals}

In this appendix, we interpret our structure of private information using the results of Athey and Levin (2001).

We first argue that the assumption that signals' marginal distribution is uniform on $[0,1]$ is without loss of generality. To see why, suppose a signal $X$ with marginal distribution $F$ strictly increasing (see Lehmann, 1988 and Athey and Levin, 2001 for the general case), and define $Z \equiv$ $F(X)$. Certainly, $Z$ has the same information content as $X$ since it is a monotone transformation, and it is easy to show that $Z$ has a uniform distribution on $[0,1]$.

Next, we interpret our assumption that $\frac{\partial E\left[V_{i} \mid X_{i}^{\eta}=x\right]}{\partial x}>0, V_{i} \in\left\{Q_{i}, T_{i}\right\}$. Let $R$ be the set of bounded-measurable linear increasing functions $g: \Omega \rightarrow \mathbb{R}$, and $U^{R}$ be the set of functions

\footnotetext{
20 For instance, suppose that each bidder's value is the sum of a private and a common value. In a monotone equilibrium, the bid of a bidder who only observes the private value is perfectly informative of her private value and not informative of the common value. However, the bid of a bidder who observes both the common value and the private value is informative of both but, in general, we do not expect it to be perfectly informative of the private value.
} 
$u: A \times \Omega \subset \mathbb{R}^{2} \rightarrow \mathbb{R}, A$ compact, such that $r(w)=u\left(a^{\prime}, w\right)-u(a, w) \in R$ for any $a^{\prime}>a$. Note that under our assumptions, the expected payoffs of a bidder who bids $a$ in an auction game conditional on either $Q_{i}=w$ or $T_{i}=w$ and for some fixed strategies of the other bidders belongs to $U^{R}$. Thus, next lemma shows that our assumption that $\frac{\partial E\left[V_{i} \mid X_{i}^{\eta}=x\right]}{\partial x}>0$ for $V_{i} \in\left\{Q_{i}, T_{i}\right\}$ ensures that there exists a selection of the best response correspondence which is increasing.

Lemma 6. Let $X$ and $V$ two random variables, where $X$ has a marginal uniform distribution on $[0,1]$. If $E[V \mid X=x]$ is increasing in $x$, there exists a selection of $\arg \max _{a \in A} E[u(a, V) \mid X=x]$, for any $u \in U^{R}$, which is also increasing in $x$.

Proof. Lemma 1 in Athey and Levin (2001) says that a sufficient condition for the existence of an increasing selection of $\arg \max _{a \in A} E[u(a, V) \mid X=x]$ for any $u \in U^{R}$ is that for any $x^{\prime}>x$, $\alpha E[V \mid X=x]+\beta \geqslant 0$ implies $\alpha E\left[V \mid X=x^{\prime}\right]+\beta \geqslant 0$ for any $\alpha>0$ and any $\beta$, this is that $E[V \mid X=x] \geqslant \frac{-\beta}{\alpha}$ implies $E\left[V \mid X=x^{\prime}\right] \geqslant \frac{-\beta}{\alpha}$. This holds true if and only if $E[V \mid X=x] \leqslant$ $E\left[V \mid X=x^{\prime}\right]$.

Finally, we interpret the order of the family $\left\{X_{i}^{\eta}\right\}_{\eta \in[0,1]}$. To do so, we follow the approach of Athey and Levin (2001). They identify more informative signals with more valuable signals for a given set of decision problems, in our case $U^{R}$.

Definition. Let $X, X^{\prime}$ and $V$ be three random variables such that the marginal distributions of $X$ and $X^{\prime}$ are uniform on $[0,1]$, and $E[V \mid X=x]$ and $E\left[V \mid X^{\prime}=x\right]$ are increasing in $x$. We say that $X$ is more informative of $V$ than signal $X^{\prime}$ if and only if,

$$
E\left[\max _{a \in A} E[u(a, V) \mid X]\right] \geqslant E\left[\max _{a \in A} E\left[u(a, V) \mid X^{\prime}\right]\right], \quad \text { for any } u \in U^{R} .
$$

Lemma 7. Let $X, X^{\prime}$ and $V$ be as in the former definition. $X$ is more informative of $V$ than $X^{\prime}$ if and only if $E[V \mid X \leqslant x] \leqslant E\left[V \mid X^{\prime} \leqslant x\right]$ (or equivalently ${ }^{21} E[V \mid X \geqslant x] \geqslant E\left[V \mid X^{\prime} \geqslant x\right]$ ) for any $x \in[0,1]$.

Proof. Direct application of Theorem 1 in Athey and Levin (2001) noting that for $X$ uniform, their function $T$ is $T(x)=x$, and our definition of the set $R$ makes their condition $C$ direct.

Lemma 8. If $\frac{\partial E\left[V_{i} \mid X^{\eta}=x\right]}{\partial x}\left(V_{i} \in\left\{Q_{i}, T_{i}\right\}\right)$ is strictly increasing in $\eta$ for any $x \in[0,1]$, then $E\left[V_{i} \mid X^{\eta} \leqslant x\right]$ is strictly decreasing (and $E\left[V_{i} \mid X^{\eta} \geqslant x\right]$ strictly increasing) in $\eta$ for any $x \in$ $(0,1)$.

Proof. To prove our lemma, we show that $\Phi(x) \equiv x\left(E\left[V_{i} \mid X_{i}^{\eta} \leqslant x\right]-E\left[V_{i} \mid X_{i}^{\eta^{\prime}} \leqslant x\right]\right)<0$ for $\eta>\eta^{\prime}$ and $x \in(0,1)$. A sufficient condition is that $\Phi(0)=0, \Phi(1)=0$, and $\Phi^{\prime \prime}(x)>0$. That $\Phi(0)=0$ is straightforward. $\Phi(1)=0$ because $E\left[V_{i} \mid X_{i}^{\eta} \leqslant 1\right]=E\left[V_{i}\right]$. Finally, note that $x E\left[V_{i} \mid X_{i}^{\eta} \leqslant x\right]=x E\left[E\left[V_{i} \mid X_{i}^{\eta}\right] X_{i}^{\eta} \leqslant x\right]=\int_{0}^{x} E\left[V_{i} \mid X_{i}^{\eta}=\tilde{x}\right] \mathrm{d} \tilde{x}$, and hence, $\Phi^{\prime \prime}(x)=$ $\frac{\partial E\left[V_{i} \mid X_{i}^{\eta}=x\right]}{\partial x}-\frac{\partial E\left[V_{i} \mid X_{i}^{\eta^{\prime}}=x\right]}{\partial x}>0$.

21 This is because by the law of iterated expectations $E[V]=x E[V \mid X \leqslant x]+(1-x) E[V \mid X \geqslant x]$. 


\section{Corollary 3.}

- If $\eta>\eta^{\prime}$ then $X_{i}^{\eta}$ is more informative of $Q_{i}$ than $X_{i}^{\eta^{\prime}}$ under the $Q$-information assumption.

- If $\eta>\eta^{\prime}$ then $X_{i}^{\eta}$ is more informative of $T_{i}$ than $X_{i}^{\eta^{\prime}}$ under the T-information assumption.

Thus, our two assumptions to order $\left\{X_{i}^{\eta}\right\}_{\eta}$, the T-information and the Q-information assumptions, are simply a refinement of the concept of more informative signals introduced by Athey and Levin (2001). This refinement was already proposed by Hagedorn (2004) to study the value of information in auctions. Ganuza and Penalva-Zuasti (2006) also use a concept of information called precision which corresponds to our refinement. They also show how our refinement corresponds to the concept of dispersion by Bickel and Lehmann (1976). Note, however, that our refinement only plays a small role in our analysis. It enters in the comparison of the incentives to acquire information only through Lemmas 1 and 2. In Lemma 1, we only need that $\mu(x, \eta)$ is increasing in $\eta$, which can also be derived from Athey and Levin's definition by Lemma 7, at least for $x$ close 1. Thus, Athey and Levin's definition should also imply a version of Lemma 1, at least for $n$ large enough. In Lemma 2, our refinement is not necessary as we only use it through Lemma 8 whose consequences hold also true for Athey and Levin's definition by Lemma 7. Our efficiency and revenue comparison also make use of our refinement through Lemmas 4 and 5, though it is only necessary in Lemma 5 since Lemma 4 only uses the implications of Lemma 7. Finally, our refinement is also used to show that $\frac{\Delta U^{a}}{\Delta \eta}\left(\eta_{d}, \eta\right)>0, a \in\{O, S\}$, in Proposition 3, but this result only plays a small role in our analysis of existence of equilibrium in Proposition 4.

Finally, we include an additional result that relates our application of the concept of more informative signals by Athey and Levin to the concepts of second order stochastic dominance and mean preserving spreads, see Rothschild and Stiglitz (1970).

Lemma 9. The signal $X^{\eta}$ is more informative of $V$ than the signal $X^{\eta^{\prime}}$ if and only if the distribution of $E\left[V \mid X^{\eta^{\prime}}\right]$ second order stochastically dominates the distribution of $E\left[V \mid X^{\eta}\right]$, this is, if and only if the distribution of $E\left[V \mid X^{\eta}\right]$ is a mean preserving spread of the distribution of $E\left[V \mid X^{\eta^{\prime}}\right]$.

Proof. Let $\Psi_{\eta}(x) \equiv E\left[V \mid X^{\eta}=x\right]$ for $x \in[0,1]$. Under our assumptions $\Psi_{\eta}$ is strictly increasing and thus invertible. Using this notation, Lemma 7 implies that to say that $X^{\eta}$ is more informative of $V$ than $X^{\eta^{\prime}}$ is equivalent to:

$$
\int_{0}^{x} \Psi_{\eta}(\tilde{x}) \mathrm{d} \tilde{x} \leqslant \int_{0}^{x} \Psi_{\eta^{\prime}}(\tilde{x}) \mathrm{d} \tilde{x}, \quad \forall x \in[0,1] .
$$

This is because $E\left[V \mid X^{\eta} \leqslant x\right]=E\left[E\left[V \mid X^{\eta}\right] \mid X^{\eta} \leqslant x\right]=\int_{0}^{x} \Psi_{\eta}(\tilde{x}) \frac{\mathrm{d} \tilde{x}}{x}$.

Let $\Psi_{\eta}^{-1}$ be the inverse of $\Psi_{\eta}$. We also let $\Psi_{\eta}^{-1}(v)=0$ for $v<\Psi_{\eta}(0)$ and $\Psi_{\eta}^{-1}(v)=1$ for $v>\Psi_{\eta}(1)$. Note that the probability of $\left\{E\left[V \mid X^{\eta}\right] \leqslant v\right\}$ is equal to the probability of $\left\{X^{\eta} \leqslant \Psi_{\eta}^{-1}(v)\right\}$, this is $\Psi_{\eta}^{-1}(v)$. Thus, according to the characterization of Rothschild and Stiglitz (1970), to say that the distribution of $E\left[V \mid X^{\eta^{\prime}}\right]$ second order stochastically dominates the distribution of $E\left[V \mid X^{\eta}\right]$, or to say that the distribution of $E\left[V \mid X^{\eta}\right]$ is a mean preserving 
spread of the distribution of $E\left[V \mid X^{\eta^{\prime}}\right]$ is equivalent to,

$$
\int_{-\infty}^{v} \Psi_{\eta}^{-1}(\tilde{v}) \mathrm{d} \tilde{v} \geqslant \int_{-\infty}^{v} \Psi_{\eta^{\prime}}^{-1}(\tilde{v}) \mathrm{d} \tilde{v}, \quad \forall v \in \mathbb{R}
$$

Next, note that using integration by parts we have that,

$$
\int_{0}^{x} \Psi_{\eta}(\tilde{x}) \mathrm{d} \tilde{x}=x \Psi_{\eta}(x)-\int_{0}^{x} \tilde{x} \mathrm{~d} \Psi_{\eta}(\tilde{x})
$$

The change of variable $\Psi_{\eta}(\tilde{x})=\tilde{v}$ in the second integral gives us,

$$
\int_{0}^{x} \Psi_{\eta}(\tilde{x}) \mathrm{d} \tilde{x}=x \Psi_{\eta}(x)-\int_{-\infty}^{\Psi_{\eta}(x)} \Psi_{\eta}^{-1}(\tilde{v}) \mathrm{d} \tilde{v}
$$

We can also apply the above equation to $\eta^{\prime}$ to get:

$$
\int_{0}^{x} \Psi_{\eta^{\prime}}(\tilde{x}) \mathrm{d} \tilde{x}=x \Psi_{\eta^{\prime}}(x)-\int_{-\infty}^{\Psi_{\eta^{\prime}}(x)} \Psi_{\eta^{\prime}}^{-1}(\tilde{v}) \mathrm{d} \tilde{v}
$$

Thus, ${ }^{22}$

$$
\begin{aligned}
& \int_{0}^{x}\left(\Psi_{\eta^{\prime}}(\tilde{x})-\Psi_{\eta}(\tilde{x})\right) \mathrm{d} \tilde{x} \\
& \quad=x\left(\Psi_{\eta^{\prime}}(x)-\Psi_{\eta}(x)\right)+\int_{-\infty}^{\Psi_{\eta}(x)}\left(\Psi_{\eta}^{-1}(\tilde{v})-\Psi_{\eta^{\prime}}^{-1}(\tilde{v})\right) \mathrm{d} \tilde{v}-\int_{\Psi_{\eta}(x)}^{\Psi_{\eta^{\prime}}(x)} \Psi_{\eta^{\prime}}^{-1}(\tilde{v}) \mathrm{d} \tilde{v} .
\end{aligned}
$$

This is,

$$
\int_{0}^{x}\left(\Psi_{\eta^{\prime}}(\tilde{x})-\Psi_{\eta}(\tilde{x})\right) \mathrm{d} \tilde{x}=\int_{-\infty}^{\Psi_{\eta}(x)}\left(\Psi_{\eta}^{-1}(\tilde{v})-\Psi_{\eta^{\prime}}^{-1}(\tilde{v})\right) \mathrm{d} \tilde{v}+\int_{\Psi_{\eta}(x)}^{\Psi_{\eta^{\prime}}(x)}\left(x-\Psi_{\eta^{\prime}}^{-1}(\tilde{v})\right) \mathrm{d} \tilde{v} .
$$

This proves that Eq. (12) implies Eq. (11) as required for the "if" part of the lemma since $\int_{\Psi_{\eta}(x)}^{\Psi_{\eta^{\prime}}(x)}\left(x-\Psi_{\eta^{\prime}}^{-1}(\tilde{v})\right) \mathrm{d} \tilde{v}$ is non-negative.

The proof of the "only if" part is symmetric. In this case, we have that,

$$
\int_{-\infty}^{v}\left(\Psi_{\eta}^{-1}(\tilde{v})-\Psi_{\eta^{\prime}}^{-1}(\tilde{v})\right) \mathrm{d} \tilde{v}=\int_{0}^{\Psi_{\eta^{\prime}}^{-1}(v)}\left(\Psi_{\eta^{\prime}}(\tilde{x})-\Psi_{\eta}(\tilde{x})\right) \mathrm{d} \tilde{x}+\int_{\Psi_{\eta^{\prime}}^{-1}(v)}^{\Psi_{\eta}^{-1}(v)}\left(v-\Psi_{\eta}(\tilde{x})\right) \mathrm{d} \tilde{x} .
$$

22 We adopt the convention that $\int_{v}^{v^{\prime}} \lambda(\tilde{v}) \mathrm{d} \tilde{v}=-\int_{v^{\prime}}^{v} \lambda(\tilde{v}) \mathrm{d} \tilde{v}$ for $v^{\prime}<v$. 
This proves that Eq. (11) implies Eq. (12) as required for the "only if" part of the lemma since $\int_{\Psi_{\eta^{\prime}}^{-1}(v)}^{\Psi_{\eta}^{-1}(v)}\left(v-\Psi_{\eta}(\tilde{x})\right) \mathrm{d} \tilde{x}$ is non-negative.

\section{A.2. Proofs}

Proof of Proposition 1. In this proof we check that bidders do not have incentives to deviate ex post, i.e. conditional on the vector of bidder's types. We only include the proof for the deviating bidder. The proof is similar for non-deviating bidders.

The expected value of a deviating bidder conditional on a vector of bidders' types $\left(x_{1}, x_{2}, \ldots\right.$, $\left.x_{n}\right) \in(0,1)^{n}$ is equal to:

$$
E\left[T_{d}+\sum_{j=1}^{n} Q_{j} \mid X_{d}^{\eta_{d}}=x_{d},\left\{X_{j}^{\eta}=x_{j}\right\}_{j \neq d}\right],
$$

whereas the price, which is fixed by the highest bid of the non-deviating bidders, say Bidder $i$, is equal to

$$
E\left[T_{i}+\sum_{j=1}^{n} Q_{j} \mid X_{d}^{\eta_{d}}=\hat{\phi}_{O}^{-1}\left(x_{i}\right),\left\{X_{j}^{\eta}=x_{j}\right\}_{j \neq d}\right],
$$

assuming that all the bidders but the deviating bidder follow our proposed strategies.

The definition of $\phi_{O}$ in Eq. (1) implies that the above expected value and price are equal for any $\left(x_{d}, x_{i}\right) \in[0,1]^{2}$ in the graph of $\phi_{O}$. Moreover, to the right of the graph of $\phi_{O}$ the expected value is greater than the price, whereas to the left is less because an increase in $x_{d}$ increases the expected value but not the price. Finally, in the set $\left\{\left(x_{d}, x_{i}\right): x_{d} \in[0,1], x_{i} \in\left(\phi_{O}(1), 1\right]\right\}$, the price is greater than the expected value whereas in $\left\{\left(x_{d}, x_{i}\right): x_{d} \in[0,1], x_{i} \in\left[0, \phi_{O}(0)\right)\right\}$ happens the opposite. This is because an increase in $x_{i}$ above $\phi_{1}(1)$ or below $\phi_{1}(1)$, increases the price more than the expected value. Hence, our proposed strategy ensures the bidder that she wins whenever the expected value is above the price and loses otherwise. Consequently, she cannot gain by deviating.

Proof of Proposition 2. First, we simplify Eq. (6). Second, we show that any solution to Eq. (6) characterizes an equilibrium in strictly increasing bid functions if and only if it satisfies certain conditions. Third, we construct a solution that satisfies the equilibrium conditions and that generates the greatest efficiency among all the solutions that verify the equilibrium conditions.

Lemma 10. A strictly increasing and continuous function $\phi$ belongs to $\Gamma$ if and only if it is the solution to the following differential equation:

$$
\begin{aligned}
& \phi^{\prime}(x)(n-2) x\left(E\left[T_{d} \mid X_{d}^{\eta_{d}}=x\right]-E\left[T_{i} \mid X_{i}^{\eta}=\phi(x)\right]+\mu\left(x, \eta_{d}\right)-\mu(\phi(x), \eta)\right) \\
& \quad=\phi(x)\left(E\left[T_{i} \mid X_{i}^{\eta}=\phi(x)\right]-E\left[T_{d} \mid X_{d}^{\eta_{d}}=x\right]\right) .
\end{aligned}
$$

Proof. The law of iterated expectations implies that the right-hand side of Eq. (6) is equal to,

$$
(1-\rho(x)) E\left[T_{i}+\sum_{j=1}^{n} Q_{j} \mid X_{i}^{\eta}=\phi(x), \max \left\{\left\{X_{j}^{\eta}\right\}_{j \neq d, i}\right\}=\phi(x), X_{d}^{\eta_{d}} \leqslant x\right]
$$




$$
+\rho(x) E\left[T_{i}+\sum_{j=1}^{n} Q_{j} \mid X_{i}^{\eta}=\phi(x), \max \left\{\left\{X_{j}^{\eta}\right\}_{j \neq d, i}\right\} \leqslant \phi(x), X_{d}^{\eta_{d}}=x\right],
$$

where

$$
\rho(x) \equiv \operatorname{Pr}\left\{X_{d}^{\eta_{d}}=x \mid \max \left\{\left\{X_{j}^{\eta}\right\}_{j \neq d, i} \cup\left\{\phi\left(X_{d}^{\eta_{d}}\right)\right\}\right\}=\phi(x)\right\} .
$$

Using this fact, we can rewrite Eq. (6) as follows after some algebra:

$$
\begin{aligned}
& (1-\rho(x))\left(E\left[T_{d} \mid X_{d}^{\eta_{d}}=x\right]-E\left[T_{i} \mid X^{\eta}=\phi(x)\right]+\mu\left(x, \eta_{d}\right)-\mu(\phi(x), \eta)\right) \\
& \quad=\rho(x)\left(E\left[T_{i} \mid X^{\eta}=\phi(x)\right]-E\left[T_{d} \mid X_{d}^{\eta_{d}}=x\right]\right),
\end{aligned}
$$

which implies the lemma since for $\phi$ strictly increasing and continuous,

$$
\rho(x)=\frac{\phi(x)^{n-2}}{\phi(x)^{n-2}+x(n-2) \phi(x)^{n-3} \phi^{\prime}(x)}=\frac{\phi(x)}{\phi(x)+x(n-2) \phi^{\prime}(x)},
$$

almost everywhere.

Certainly, for any $\phi \in \Gamma$, the corresponding function $b_{d}^{S}$ is strictly increasing. Moreover:

Lemma 11. For any $\phi \in \Gamma$, the corresponding function $b^{S}$ is strictly increasing if and only if $\hat{\phi}(1) \geqslant \hat{\phi}_{*}(1)$.

Proof. That $b^{S}$ is increasing in $[0, \hat{\phi}(0))$, around $\hat{\phi}(0)$ and in $(\hat{\phi}(1), 1]$ is straightforward from its definition, and in $(\hat{\phi}(0), \hat{\phi}(1))$, is a consequence of $b^{S}(x)=b_{d}^{S}\left(\phi^{-1}(x)\right)$ by definition of $\phi^{-1}$ in Eq. (6), and the fact that $\phi$ is increasing and hence $b_{d}^{S}(x)$ and $\hat{\phi}^{-1}$. It only remains to be shown that $b^{S}$ is increasing around $x=\hat{\phi}(1)$ if and only if $\hat{\phi}(1) \geqslant \hat{\phi}_{*}(1)$. Certainly, this is only an issue if $\hat{\phi}(1)<1$. In this case, $b^{S}(\hat{\phi}(1))=b_{d}^{S}(1)$ by definition of $\hat{\phi}$. Hence,

$$
\begin{aligned}
& b^{S}(\hat{\phi}(1))-\inf _{x>\hat{\phi}(1)}\left\{b^{S}(x)\right\}=b_{d}^{S}(1)-\inf _{x>\hat{\phi}(1)}\left\{b^{S}(x)\right\} \\
& \quad=E\left[T_{d} \mid X_{d}^{\eta_{d}}=1\right]-E\left[T_{i} \mid X_{i}^{\eta}=\hat{\phi}(1)\right]+\mu\left(1, \eta_{d}\right)-\mu(\hat{\phi}(1), \eta),
\end{aligned}
$$

which by definition of $\phi_{*}(1)$, see Eq. (10), is non-positive if and only if $\hat{\phi}(1) \geqslant \hat{\phi}_{*}(1)$ as desired.

Lemma 12. For any function $\phi \in \Gamma$ that satisfies the condition in Lemma 11, it is an equilibrium that the deviating bidder uses the corresponding function $b_{d}^{S}$ and the non-deviating bidders the corresponding bid function $b^{S}$ if and only if $\hat{\phi}^{-1}(1) \geqslant \hat{\phi}_{O}^{-1}(1)$.

Proof. This proof has several similarities with the proof of Proposition 1. It differs in that we do not compute the incentives to deviate conditional on the vector of bidders' types. Instead, we condition on the maximum bid of the other bidders, i.e. the price if the bidder wins. This proof generalizes the corresponding proof of Milgrom and Weber (1982a) to our asymmetric model.

We start with the incentives to deviate of the deviating bidder assuming that all the nondeviating bidders follow our proposed strategy. Under this assumption, to condition on the price is equivalent to condition on the maximum type of the non-deviating bidders equal to the type 
who submits that price, say $x$. The expected value of a deviating bidder with type $x_{d}$ conditional on the former event is equal to,

$$
E\left[T_{d}+\sum_{j=1}^{n} Q_{j} \mid X_{d}^{\eta_{d}}=x_{d}, \max \left\{X_{j}^{\eta}\right\}_{j \neq d}=x\right],
$$

and the price, if she wins, the bid of the non-deviating bidder with type $x$,

$$
E\left[T_{i}+\sum_{j=1}^{n} Q_{j} \mid X_{i}^{\eta}=\max \left\{\left\{X_{j}^{\eta}\right\}_{j \neq d, i} \cup\left\{\hat{\phi}\left(X_{d}^{\eta_{d}}\right)\right\}\right\}=x\right] .
$$

The remaining of the proof is almost identical to the proof of Proposition 1. It only differs in that we use Eq. (6) instead of Eq. (1).

Next, we study the incentives to deviate of a non-deviating bidder, say Bidder $i$, with type $x_{i}$ assuming that all the other bidders follow the proposed strategies. Consider first the case in which the highest of the other bidders' bids is above $b^{S}(1)$. In this case, the highest bid is submitted by the deviating bidder and her type, say $x_{d}$, is greater than $\hat{\phi}^{-1}(1)$. If Bidder $i$ follows our proposed strategy, she loses. Suppose, however, that she deviates and wins. Her conditional expected value is equal to $E\left[T_{i}+\sum_{j=1}^{n} Q_{j} \mid X_{d}^{\eta_{d}}=x_{d}, X_{i}^{\eta}=1\right]$, and the price she pays the deviating bidder's bid. This is,

$$
E\left[T_{d}+\sum_{j=1}^{n} Q_{j} \mid X_{d}^{\eta_{d}}=x_{d}, \max \left\{X_{j}^{\eta}\right\}_{j \neq d}=1\right] .
$$

Thus, the difference between expected value and price is equal to $E\left[T_{i} \mid X_{i}^{\eta}=1\right]-E\left[T_{d} \mid X_{d}^{\eta_{d}}=\right.$ $x_{d}$ ]. By definition of $\phi_{O}^{-1}(1)$, see Eq. (2), this expression is non-positive for any $x_{d}>\hat{\phi}^{-1}(1)$ if and only $\hat{\phi}^{-1}(1) \geqslant \hat{\phi}_{O}^{-1}(1)$ as required in the lemma.

Suppose now that the highest of the other bidders' bids, say $b$, is between $b^{S}(0)$ and $b^{S}(1)$. Then, we can deduce that $\max \left\{\left\{b^{S}\left(X_{j}^{\eta}\right)\right\}_{j \neq d, i} \cup\left\{b^{S}\left(\hat{\phi}\left(X_{d}^{\eta_{d}}\right)\right)\right\}\right\}=b$, and hence that $\max \left\{\left\{X_{j}^{\eta}\right\}_{j \neq d, i} \cup\left\{\hat{\phi}\left(X_{d}^{\eta_{d}}\right)\right\}\right\}=\left(b^{S}\right)^{-1}(b)$. Thus, the conditional expected value of Bidder $i$ is equal to

$$
E\left[T_{i}+\sum_{j=1}^{n} Q_{j} \mid X_{i}^{\eta}=x_{i}, \max \left\{\left\{X_{j}^{\eta}\right\}_{j \neq d, i} \cup\left\{\hat{\phi}\left(X_{d}^{\eta_{d}}\right)\right\}\right\}=\left(b^{S}\right)^{-1}(b)\right],
$$

and the price if she wins is $b$ which is equal to

$$
E\left[T_{i}+\sum_{j=1}^{n} Q_{j} \mid X_{i}^{\eta}=\max \left\{\left\{X_{j}^{\eta}\right\}_{j \neq d, i} \cup\left\{\hat{\phi}\left(X_{d}^{\eta_{d}}\right)\right\}\right\}=\left(b^{S}\right)^{-1}(b)\right] .
$$

The difference between the above price and expected value is positive if and only if $x_{i} \geqslant$ $\left(b^{S}\right)^{-1}(b)$, this is when Bidder $i$ wins if she follows our proposed strategy. This concludes the proof since the highest of the other bidders' bids cannot be less than $b^{S}(0)$.

Finally, we show that there exists a function $\phi \in \Gamma$ that verifies the required conditions. Note that the second item of the lemma implies that any other equilibrium characterized by the bid functions $\left(b^{S}, b_{d}^{S}\right)$ that correspond to a $\phi \in \Gamma$ implements a less efficient allocation. This is because the efficient allocation is characterized by $\phi_{O}$. 


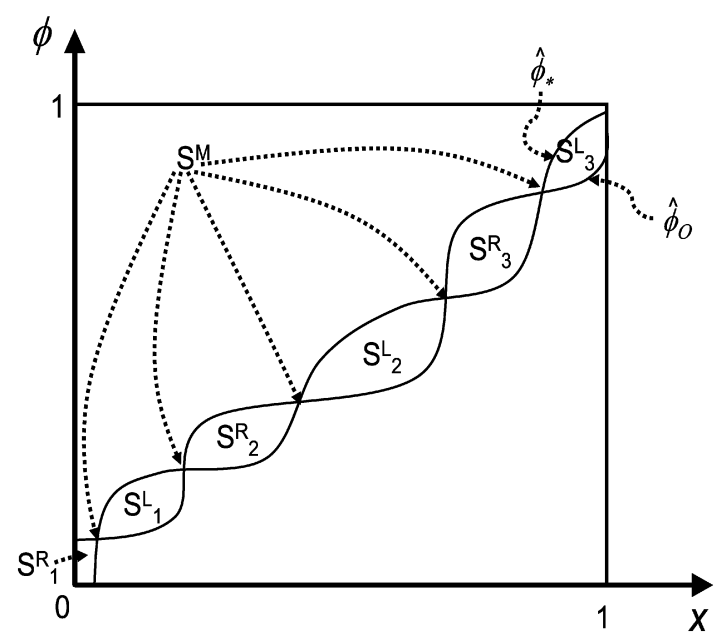

Fig. 2. Illustration of $S^{M},\left\{S_{l}^{L}\right\}$ and $\left\{S_{l}^{R}\right\}$ for some arbitrary $\hat{\phi}_{O}$ and $\hat{\phi}_{*}$.

Lemma 13. There exists a $\phi_{S, n} \in \Gamma$ that satisfies the conditions in Lemmas 11 and 12 and it is such that:

- $\hat{\phi}^{-1}(1)<\hat{\phi}_{*}^{-1}(1)$, when $\hat{\phi}_{O}^{-1}(1)<\hat{\phi}_{S, n}^{-1}(1)$; and $\hat{\phi}(1)<\hat{\phi}_{*}(1)$, when $\hat{\phi}_{O}(1)<\hat{\phi}_{S, n}(1)$.

- There is no other function in $\tilde{\phi} \in \Gamma$ verifying the conditions in Lemmas 11 and 12 such that $\tilde{\phi}(x)$ belongs to the interior of the convex hull of $\left\{\hat{\phi}_{S, n}(x), \hat{\phi}_{O}(x)\right\}$ for some $x \in[0,1]$.

Proof. We prove the lemma by construction. Let $S^{M} \equiv\left\{(x, \phi): \phi=\phi_{O}(x)=\phi_{*}(x)\right\}, S^{L} \equiv$ $\left\{(x, \phi): \phi \in\left(\hat{\phi}_{O}(x), \hat{\phi}_{*}(x)\right)\right\}$, and $S^{R} \equiv\left\{(x, \phi): \phi \in\left(\hat{\phi}_{*}(x), \hat{\phi}_{O}(x)\right)\right\}$. Let also $\left\{S_{l}^{L}\right\}$ and $\left\{S_{l}^{R}\right\}$ be the collections of largest open-connected sets, in which $S^{L}$ and $S^{R}$, respectively, can be split, see Fig. 2.

In $S^{M}$, we let $\phi_{S, n}(x)$ be equal to $\phi_{O}(x)$ which also implies that it is equal to $\phi_{*}(x)$, and thus it satisfies trivially Eq. (13) and the conditions of the lemma. To construct our solution in $S^{R}$ and $S^{L}$, let

$$
\Phi(x, \phi) \equiv \frac{\phi}{x(n-2)} \frac{E\left[T_{i} \mid X_{i}^{\eta}=\phi\right]-E\left[T_{d} \mid X_{d}^{\eta_{d}}=x\right]}{E\left[T_{d} \mid X_{d}^{\eta_{d}}=x\right]-E\left[T_{i} \mid X_{i}^{\eta}=\phi\right]+\mu\left(x, \eta_{d}\right)-\mu(\phi, \eta)} .
$$

Note that solutions to $\phi^{\prime}(x)=\Phi(x, \phi(x))$ are also solutions to Eq. (13). In sets $S_{l}^{R}$ whose boundary does not contain points in the boundary of $[0,1]^{2}$ we define $\phi_{S, n}$ as the maximum increasing solution to $\phi^{\prime}(x)=\Phi(x, \phi(x))$ that starts at the infimum of $S_{l}^{R}$, that we denote by $\left(\underline{x}_{l}^{R}, \underline{\phi}_{l}^{R}\right)$, and ends at its supremum, that we denote by $\left(\bar{x}_{l}^{R}, \bar{\phi}_{l}^{R}\right)$. Note that this solution is a continuation of the solution $\phi_{S, n}$ that we have defined in $S^{M}$ because $\left(\underline{x}_{l}^{R}, \underline{\phi}_{l}^{R}\right)$ and $\left(\bar{x}_{l}^{R}, \bar{\phi}_{l}^{R}\right)$ belong to $S^{M}$ since $\phi_{O}$ and $\phi_{*}$ are strictly increasing. We show in four steps that the required solution exists.

Step 1. There exists a unique solution to $\phi^{\prime}(x)=\Phi(x, \phi(x))$ that passes by any point $\left(x_{0}, \phi_{0}\right) \in$ $S_{l}^{R}$. This solution is strictly increasing. 
Proof. The first claim is a direct application of Coddington and Levinson (1984, Theorem 2.2, p. 10) since $\Phi$ is differentiable, and thus satisfies a Lipschitz condition. The solution is strictly increasing because the denominator and the numerator of the expression that defines $\Phi$ are strictly negative. This is because the numerator is equal to zero at $\phi=\phi_{O}(x)$ and is strictly increasing in $\phi$, whereas the denominator is equal to zero at $\phi=\phi_{*}(x)$ and is strictly decreasing in $\phi$.

Step 2. The solutions to $\phi^{\prime}(x)=\Phi(x, \phi(x))$ in $S_{l}^{R}$ do not cross and converge to $\left(\bar{x}_{l}^{R}, \bar{\phi}_{l}^{R}\right)$.

Proof. That they do not cross is a consequence of Step 1. The convergence to $\left(\bar{x}_{l}^{R}, \bar{\phi}_{l}^{R}\right)$ is because, as we show next, any solution to our differential equation cannot cross neither $\phi_{O}$ nor $\phi_{*}$ as it is continued to the right. First, it cannot cross $\phi_{O}$ because the slope of $\phi_{O}$ is bounded away from zero, which can be proved applying the implicit function theorem to Eq. (2), and the slope of any solution tends to zero, as it approaches the graph of $\phi_{O}$ from below by definition of $\Phi$. And second, the solution cannot cross $\phi_{*}$ because the slope of $\phi_{*}$ has derivative bounded from above, which can be proved applying the implicit function theorem to Eq. (10), and the slope of any solution tends to infinity, as it approaches the graph of $\phi_{*}$ from above by definition of $\Phi$.

Step 3. Take any $\left(x_{0}, \phi_{0}\right)$ in the boundary of $S_{l}^{R}$ and either in the graph of $\phi_{O}$ or in the graph of $\phi_{*}$ but not in both. Then, there exists a solution to $\phi^{\prime}(x)=\Phi(x, \phi(x))$ that passes by $\left(x_{0}, \phi_{0}\right)$ and which continues to the interior of $S_{l}^{R}$.

Proof. For points in the graph of $\phi_{O}$, existence follows from an identical argument as in Step 1. Next note that the solution continues towards the interior of $S_{l}^{R}$ because $\phi_{O}^{\prime}\left(x_{0}\right)>0$ by application of the implicit function theorem to Eq. (2) and our solution has zero slope at $\left(x_{0}, \phi_{0}\right)$ since $\Phi\left(x_{0}, \phi_{0}\right)=0$ when $\phi_{0}=\phi_{O}(x) \neq \phi_{*}(x)$ and $x \neq 0$. For points in the graph of $\phi_{*}$, note that we can apply the same arguments but to the inverse differential equation $\psi^{\prime}(\phi)=\frac{1}{\Phi(\psi(\phi), \phi)}$ to show that there exists a solution $\psi$ that passes by $\left(\phi_{0}, x_{0}\right)$ and that continues towards the interior of $S_{l}^{R}$. Certainly, this solution is strictly increasing in $S_{l}^{R}$ and thus invertible. Hence, the inverse of $\psi$ is a solution to our original differential equation with the required properties.

Denote by $\Gamma\left(S_{l}^{R}\right)$ the set of continuous increasing solutions to $\phi^{\prime}(x)=\Phi(x, \phi(x))$ in $S_{l}^{R}$ whose graph starts at $\left(\underline{x}_{l}^{R}, \phi_{l}^{R}\right)$ and ends at $\left(\bar{x}_{l}^{R}, \bar{\phi}_{l}^{R}\right)$. Let also $\left\{\left(x_{\xi}, \phi_{O}\left(x_{\xi}\right)\right)\right\}$, where $x_{\xi} \rightarrow \underline{x}_{l}^{R}$, be a decreasing sequence of initial conditions that defines a sequence of solutions to our differential equation. By Step 3 the sequence of solutions exists and by Step 2 is decreasing and thus pointwise convergent to a limit function that we denote by $\bar{\phi}$. We shall make $\phi_{S, n}$ equal to $\bar{\phi}$ in these sets.

Step 4. $\bar{\phi} \in \Gamma\left(S_{l}^{R}\right)$ and $\bar{\phi}(x) \geqslant \tilde{\phi}(x), \forall x$, for any $\tilde{\phi} \in \Gamma\left(S_{l}^{R}\right)$.

Proof. Note first that $\bar{\phi}$ is an increasing function whose graph starts at $\left(\underline{x}_{l}^{R}, \underline{\phi}_{l}^{R}\right)$ and ends at $\left(\bar{x}_{l}^{R}, \bar{\phi}_{l}^{R}\right)$ by definition and Steps 2 and 3. They also imply that $\bar{\phi}(x) \geqslant \tilde{\phi}(x), \forall x$, for any $\tilde{\phi} \in$ $\Gamma\left(S_{l}^{R}\right)$ and $\bar{\phi}(x)<\phi_{O}(x)$ for any $x \in\left(\underline{x}_{l}^{R}, \bar{x}_{l}^{R}\right)$. We shall show that it is also true that $\bar{\phi}(x)>$ $\phi_{*}(x)$.

Let $\underline{\phi}($.$) be the point-wise limit of a sequence of solutions to our differential equation with$ initial conditions $\left\{\left(x_{\xi}, \phi_{*}\left(x_{\xi}\right)\right)\right\}$ where $x_{\xi} \rightarrow \underline{x}_{l}^{R}$. Similar arguments as for $\bar{\phi}($.$) show that \underline{\phi}($.$) is$ 
well defined. Moreover, by definition and Step $2 \bar{\phi}(x) \geqslant \underline{\phi}(x)>\phi_{*}(x)$ for any $x \in\left(\underline{x}_{l}^{R}, \bar{x}_{l}^{R}\right)$ as required.

Next note that for any $x \in\left(\underline{x}_{l}^{R}, \bar{x}_{l}^{R}\right),(x, \bar{\phi}(x))$ defines a unique solution to our differential equation by Step 1 . Moreover, by definition of $\bar{\phi}$ and Step 2 this solution remains weakly below $\bar{\phi}$ for any $\tilde{x} \in\left(\underline{x}_{l}^{R}, \bar{x}_{l}^{R}\right)$. Thus, $\phi_{(1)}\left(x_{(1)}\right)-\phi_{(2)}\left(x_{(1)}\right) \geqslant 0$, and $\phi_{(1)}\left(x_{(2)}\right)-\phi_{(2)}\left(x_{(2)}\right) \leqslant 0$ where $\phi_{(1)}($.$) and \phi_{(2)}($.$) denote the solutions passing by \left(x_{(1)}, \bar{\phi}\left(x_{(1)}\right)\right)$ and $\left(x_{(2)}, \bar{\phi}\left(x_{(2)}\right)\right)$ respectively, for $x_{(1)}, x_{(2)} \in\left(\underline{x}_{l}^{R}, \bar{x}_{l}^{R}\right)$. Hence, by continuity of $\phi_{(1)}$ and $\phi_{(2)}$ and Step 2, we have that $\phi_{(1)}(x)=$ $\phi_{(2)}(x)$ for $x \in\left[x_{(1)}, x_{(2)}\right]$, and since $x_{(1)}$ and $x_{(2)}$ were arbitrary numbers in $\left(\underline{x}_{l}^{R}, \bar{x}_{l}^{R}\right)$, we can conclude that $\bar{\phi}$ is a solution to our differential equation.

Note that $\bar{\phi}$ satisfies item 1 of Proposition 2, and that item 2 and the conditions in Lemmas 11 and 12 do not apply. Finally, to see that it also satisfies item 3 note that an increase in $n$ decreases $\Phi(x, \phi)$ in $S_{l}^{R}$ and thus the vector field associated. Hence, $\bar{\phi}$ also decreases point-wise and consequently approaches $\phi_{*}$.

In sets $S_{l}^{R}$ whose boundary intersects with the boundary of $[0,1]^{2}$ the construction of our solution is similar although with some small differences. In particular, the appropriate statement of Step 2 should say that the convergence is to the supremum only if does not belong to the upper or right boundary of $[0,1]^{2}$, otherwise, we can only say that there is convergence to a point in either of those boundaries. Similarly, $\Gamma\left(S_{l}^{R}\right)$ denotes the set of continuous increasing solutions to $\phi^{\prime}(x)=\Phi(x, \phi(x))$ in $S_{l}^{R}$ that start at either the infimum of $S_{l}^{R}$ or the left or down boundary of $[0,1]^{2}$ and end at the supremum of $S_{l}^{R}$ if it is not in the right or upper boundary of $[0,1]^{2}$ or otherwise at a point in any of these two boundaries. The last difference is that in Step 3 we write for $\left(x_{0}, \phi_{0}\right) \neq(0,0)$ and $\hat{\phi}_{*}$ instead of $\phi_{*}$. We also use $\hat{\phi}_{*}$ instead of $\phi_{*}$ to define $\phi$ in the proof of Step 4. It is easy to check that the corresponding solution also satisfies all the items of Proposition 2 and the conditions in Lemmas 11 and 12.

The construction in sets $S_{l}^{L}$ is similar with only three differences: In Step 2, we extend the auxiliary solutions to the left and the solutions converge to the infimum of $S_{l}^{L}$ when $S_{l}^{L}$ does not intersect with the left or down boundary of $[0,1]^{2}$, otherwise the converge is to a point in either of both boundaries. $\Gamma\left(S_{l}^{L}\right)$ denotes the set of continuous increasing solutions to $\phi^{\prime}(x)=\Phi(x, \phi(x))$ in $S_{l}^{L}$ that start at the supremum of $S_{l}^{R}$ and end at the infimum of $S_{l}^{L}$ if it is not in the left or bottom boundary of $[0,1]^{2}$, or otherwise at a point in either of these boundaries. Note that the solutions in $\Gamma\left(S_{l}^{L}\right)$ satisfies the conditions of Lemmas 11 and 12 because these solutions start at the supremum of $S_{l}^{L}$ when it lies in the upper or right boundary of $[0,1]^{2}$. Finally, to define our solution $\bar{\phi}$ before Step 4 , we use an increasing sequence of initial conditions $\left\{\left(x_{\xi}, \phi_{O}\left(x_{\xi}\right)\right)\right\}$ if the boundary of $S_{l}^{L}$ does not intersect with the right boundary of $[0,1]^{2}$. Otherwise, we define $\bar{\phi}$ with an increasing sequence $\left\{\left(1, \phi_{\xi}\right)\right\}$ where $\phi_{\xi} \rightarrow \hat{\phi}_{*}(1)$. In either case, we define the corresponding function $\underline{\phi}$ with an increasing sequence $\left\{x_{\xi}, \hat{\phi}_{*}\left(x_{\xi}\right)\right\}$ of initial conditions for $x_{\xi}$ converging to 1 .

Similar reasons as in sets $S_{l}^{R}$ explain that the items of Proposition 2 are also satisfied in sets $S_{l}^{L}$. That the conditions of Lemmas 11 and 12 are satisfied in sets $S_{l}^{L}$ is explained above.

Proof of Lemma 1. Suppose that $\eta_{d}>\eta$, the other case is symmetric. It is easy to show that $E\left[T_{d} \mid X_{d}^{\eta_{d}}=x\right]$ is constant in $\eta_{d}$ under the Q-information assumption, which implies $\hat{\phi}_{O}(x)=x$, see Eq. (2). Next, applying, first, the law of iterated expectations and, second, integrating by parts, we have that, 


$$
\mu\left(x, \eta_{d}\right)=E\left[Q_{d} \mid X_{d}^{\eta_{d}}=x\right]-\int_{0}^{x} E\left[Q_{d} \mid X_{d}^{\eta_{d}}=\tilde{x}\right] \frac{\mathrm{d} \tilde{x}}{x}=\int_{0}^{x} \frac{\tilde{x}}{x} \frac{\partial E\left[Q_{d} \mid X_{d}^{\eta_{d}}=\tilde{x}\right]}{\partial \tilde{x}} \mathrm{~d} \tilde{x},
$$

which means that $\mu\left(x, \eta_{d}\right)$ is increasing in $\eta_{d}$ by definition of the Q-information assumption. Thus, $E\left[T_{d} \mid X_{d}^{\eta_{d}}=x\right]-E\left[T_{i} \mid X_{i}^{\eta}=x\right]+\mu\left(x, \eta_{d}\right)-\mu(x, \eta)>0$, for $x \in(0,1)$. As a consequence, $\hat{\phi}_{*}(x)>x$ by definition of $\phi_{*}$, see Eq. (10). The fact that $\hat{\phi}_{*}(x)>x$ and $\hat{\phi}_{O}(x)=x$, together with item 1 of Proposition 2 implies the lemma.

Proof of Lemma 2. Suppose that $\eta_{d}>\eta$, the other case is symmetric. By Lemma 8 in Appendix A.1, $E\left[T_{d} \mid X_{d}^{\eta_{d}}=1\right]$ strictly increases in $\eta_{d}$ under the T-information assumption. Thus, $E\left[T_{d} \mid X_{d}^{\eta_{d}}=1\right]-E\left[T_{i} \mid X_{i}^{\eta}=1\right]>0$, and as a consequence, $\phi_{O}^{-1}(1)<1$ by definition of $\phi_{O}$, see Eq. (2), and $E\left[T_{d} \mid X_{d}^{\eta_{d}}=1\right]-E\left[T_{i} \mid X_{i}^{\eta}=1\right]+\mu\left(1, \eta_{d}\right)-\mu(1, \eta)>0$ since $\mu\left(\tilde{x}, \eta_{d}\right)=\mu(\tilde{x}, \eta)$ under the T-information assumption. Moreover, $E\left[T_{d} \mid X_{d}^{\eta_{d}}=\phi_{O}^{-1}(1)\right]-$ $E\left[T_{i} \mid X_{i}^{\eta}=1\right]+\mu\left(\phi_{O}^{-1}(1), \eta_{d}\right)-\mu(1, \eta)<0$, since $E\left[T_{d} \mid X_{d}^{\eta_{d}}=\phi_{O}^{-1}(1)\right]-E\left[T_{i} \mid X_{i}^{\eta}=1\right]=0$ by definition of $\phi_{O}, \mu\left(x, \eta_{d}\right)=\mu(x, \eta)$ and $\mu\left(\tilde{x}, \eta_{d}\right)$ is increasing in $\tilde{x}$. Consequently, $\phi_{O}^{-1}(1)<$ $\phi_{*}^{-1}(1)<1$ by definition of $\phi_{*}$, see Eq. (10). An application of item 1 and 2 in Proposition 2 implies that $\phi_{O}^{-1}(1)<\phi_{S, n}^{-1}(1) \leqslant \phi_{*}^{-1}(1)<1$, which implies the lemma by continuity.

Proof of Lemma 3. This proof is an adaptation of the arguments by Myerson (1981) to our model. Let $u\left(x_{d}, \tilde{x}_{d}\right)$ be the expected utility of Bidder $d$ with type $x_{d}$ given that all other bidders use their equilibrium strategies and she submits the bid that corresponds to her equilibrium strategy when she has type $\tilde{x}_{d}$.

For any given profile of strategies, we can define a function $Q:[0,1]^{n} \rightarrow[0,1]^{n}$ and a function $P:[0,1]^{n} \rightarrow \mathbb{R}^{n}$ where $Q_{i}\left(x_{1}, \ldots, x_{n}\right)$ denotes the probability with which Bidder $i$ gets the good and $P_{i}\left(x_{1}, \ldots, x_{n}\right)$ her expected payment when the bidders submit the bids that corresponds to their equilibrium strategies when they have types $\left(x_{1}, \ldots, x_{n}\right)$. Under the conditions of the lemma, $Q_{d}\left(x_{1}, \ldots, x_{n}\right)=\mathbf{1}_{\left\{\phi\left(x_{d}\right) \geqslant \max \left\{x_{i}\right\}_{i \neq d}\right\}}$. Thus, we can write that

$$
u\left(x_{d}, \tilde{x}_{d}\right)=E\left[1_{\left\{\phi\left(\tilde{x}_{d}\right) \geqslant \max \left\{X_{i}^{\eta}\right\}_{i \neq d}\right\}}\left(T_{d}+\sum_{j=1}^{n} Q_{j}\right) \mid X_{d}^{\eta_{d}}=x_{d}\right]-p_{d}\left(\tilde{x}_{d}\right),
$$

where $p_{d}\left(\tilde{x}_{d}\right)=E\left[P_{d}\left(X_{1}^{\eta}, \ldots, X_{d-1}^{\eta}, \tilde{x}_{d}, X_{d+1}^{\eta}, \ldots, X_{n}^{\eta}\right)\right]$. By rearranging terms and noting that $E\left[1_{\left\{\phi\left(\tilde{x}_{d}\right) \geqslant \max \left\{X_{i}^{\eta}\right\}_{i \neq d}\right\}}\right]=\phi\left(\tilde{x}_{d}\right)^{n-1}$, we have that

$$
\begin{aligned}
u\left(x_{d}, \tilde{x}_{d}\right)= & E\left[1_{\left\{\phi\left(\tilde{x}_{d}\right) \geqslant \max \left\{X_{i}^{\eta}\right\}_{i \neq d}\right\}} \cdot \sum_{j \neq d} Q_{j}\right] \\
& +\phi\left(\tilde{x}_{d}\right)^{n-1} E\left[T_{d}+Q_{d} \mid X_{d}^{\eta_{d}}=x_{d}\right]-p_{d}\left(\tilde{x}_{d}\right) .
\end{aligned}
$$

Let $u^{*}\left(x_{d}\right) \equiv u\left(x_{d}, x_{d}\right)$, this is, $u^{*}\left(x_{d}\right)$ denotes the equilibrium expected utility of Bidder $d$ with type $x_{d}$. By the envelope theorem, $\frac{\partial u^{*}(x)}{\partial x}=\left.\frac{\partial u(x, \tilde{x})}{\partial x}\right|_{\tilde{x}=x}$. As a consequence,

$$
\frac{\partial u^{*}(x)}{\partial x}=\phi(x)^{n-1} \frac{\partial E\left[T_{d}+Q_{d} \mid X_{d}^{\eta_{d}}=x\right]}{\partial x},
$$


which by the fundamental theorem of calculus implies

$$
u^{*}(y)=\int_{0}^{y} \phi(x)^{n-1} \frac{\partial E\left[T_{d}+Q_{d} \mid X_{d}^{\eta_{d}}=x\right]}{\partial x} \mathrm{~d} x+u^{*}(0) .
$$

Thus, the ex ante expected utility of the deviating bidder is equal to:

$$
\begin{aligned}
& \int_{0}^{1} \int_{0}^{y} \phi(x)^{n-1} \frac{\partial E\left[T_{d}+Q_{d} \mid X_{d}^{\eta_{d}}=x\right]}{\partial x} \mathrm{~d} x \mathrm{~d} y+u^{*}(0) \\
& \quad=\int_{0}^{1} \int_{0}^{1} 1_{\{y \geqslant x\}} \phi(x)^{n-1} \frac{\partial E\left[T_{d}+Q_{d} \mid X_{d}^{\eta_{d}}=x\right]}{\partial x} \mathrm{~d} x \mathrm{~d} y+u^{*}(0) \\
& =\int_{0}^{1}\left(\int_{0}^{1} 1_{\{y \geqslant x\}} \mathrm{d} y\right) \phi(x)^{n-1} \frac{\partial E\left[T_{d}+Q_{d} \mid X_{d}^{\eta_{d}}=x\right]}{\partial x} \mathrm{~d} x+u^{*}(0) \\
& =\int_{0}^{1}(1-x) \phi(x)^{n-1} \frac{\partial E\left[T_{d}+Q_{d} \mid X_{d}^{\eta_{d}}=x\right]}{\partial x} \mathrm{~d} x+u^{*}(0) .
\end{aligned}
$$

Proof of Proposition 3. We first show that $U^{O}(\eta, \eta)=U^{S}(\eta, \eta)$. Note that $\eta_{d}=\eta$, implies that $\hat{\phi}_{O}(x)=x$, see Eq. (2), and that $\hat{\phi}_{*}(x)=x$, see Eq. (10). Thus, $\hat{\phi}_{S, n}(x)=x$ by item 1 of Proposition 2. Moreover, a deviating bidder with type zero gets zero expected utility in both auctions because her probability of winning in each auction, i.e. $\hat{\phi}_{O}(0)^{n}$ and $\hat{\phi}_{S, n}(0)^{n}$, is equal to zero since $\hat{\phi}_{O}(x)=x$ and $\hat{\phi}_{S, n}(x)=x$. We can, thus, conclude from Lemma 3 that $U^{O}(\eta, \eta)=$ $U^{S}(\eta, \eta)=\int_{0}^{1}(1-x) x^{n} \frac{\partial E\left[T_{d}+Q_{d} \mid X_{d}^{\eta}=x\right]}{\partial x} \mathrm{~d} x$.

Next, we consider the Q-information assumption. We can use a similar argument as above to show that $\eta_{d}>\eta$ implies that $U^{S}\left(\eta_{d}, \eta\right)>U^{O}\left(\eta_{d}, \eta\right)$. In particular, this implication can be deduced from Lemmas 1 and 3, from the fact that each type of a bidder gets non-negative expected utility, and from the fact that a deviating bidder with zero type gets zero expected utility in the open auction since $\hat{\phi}_{O}(0)=0$ by continuity and Lemma 1 . We can also show that $\eta_{d}<\eta$ implies $U^{S}\left(\eta_{d}, \eta\right)<U^{O}\left(\eta_{d}, \eta\right)$ with a symmetric argument. The only remarkable difference is that the reason why $\hat{\phi}_{S, n}(0)$ is equal to zero is item 1 of Proposition 2 and $\hat{\phi}_{O}(0)=$ $\hat{\phi}_{*}(0)=0$, where $\hat{\phi}_{O}(0)=0$ by continuity and Lemma 1 , and $\hat{\phi}_{*}(0)=0$ since $\mu(0, \tilde{\eta})=0$ for all $\tilde{\eta}$ by definition of $\mu$ and $E\left[T_{d} \mid X_{d}^{\eta_{d}}=x\right]=E\left[T_{i} \mid X_{i}^{\eta}=x\right]$ by the Q-information assumption, see Eq. (10). Finally, we can also deduce from Lemmas 1 and 3, and the definition of the Qinformation assumption that $U^{O}\left(\eta_{d}, \eta\right)>U^{O}(\eta, \eta)$, if $\eta_{d}>\eta$, and $U^{O}\left(\eta_{d}, \eta\right)<U^{O}(\eta, \eta)$, if $\eta_{d}<\eta$. The above results imply the first item of the lemma.

The proof of the second item is slightly different. Consider the T-information assumption. We show that $\eta_{d}>\eta$ implies $U^{O}\left(\eta_{d}, \eta\right)>U^{S}\left(\eta_{d}, \eta\right)$ for $n$ large enough by arguing that 
$\lim _{n \rightarrow \infty}\left(U^{O}\left(\eta_{d}, \eta\right)-U^{S}\left(\eta_{d}, \eta\right)\right)>0$. Lemma 3, the dominated convergence theorem and the fact that $\hat{\phi}_{O}(x)=1$ for $x \geqslant \hat{\phi}_{O}^{-1}(1)$ and $\hat{\phi}_{O}(x)<1$ otherwise, imply that:

$$
\lim _{n \rightarrow \infty} U^{O}\left(\eta_{d}, \eta\right)=\int_{\hat{\phi}_{O}^{-1}(1)}^{1}(1-x) \frac{\partial E\left[T_{d}+Q_{d} \mid X_{d}^{\eta_{d}}=x\right]}{\partial x} \mathrm{~d} x
$$

since the expected utility of the deviating bidder with a type zero tends to zero when $n$ goes to infinity. The latter because the probability that she wins, i.e. $\hat{\phi}_{O}(0)^{n}$, tends to zero. A similar argument can be applied to the sealed bid auction with the only difference that we use the monotone convergence theorem and item 3 of Proposition 2. The corresponding limit is:

$$
\lim _{n \rightarrow \infty} U^{S}\left(\eta_{d}, \eta\right)=\int_{\hat{\phi}_{S, \infty}^{-1}(1)}^{1}(1-x) \frac{\partial E\left[T_{d}+Q_{d} \mid X_{d}^{\eta_{d}}=x\right]}{\partial x} \mathrm{~d} x
$$

where $\hat{\phi}_{S, \infty}^{-1}$ (1) denotes the limit of $\hat{\phi}_{S, n}^{-1}(1)$. Lemma 2 and item 3 of Proposition 2 imply that $\hat{\phi}_{O}^{-1}(1)<\hat{\phi}_{S, \infty}^{-1}(1)$ and thus that the limit in Eq. (16) is strictly greater than the limit in Eq. (17). We show that $\eta_{d}<\eta$ implies $U^{O}\left(\eta_{d}, \eta\right)<U^{S}\left(\eta_{d}, \eta\right)$ for $n$ large enough with a similar argument, though in this case we compare $\lim _{n \rightarrow \infty} \frac{U^{O}\left(\eta_{d}, \eta\right)}{\hat{\phi}_{O}(1)^{n}}$ and $\lim _{n \rightarrow \infty} \frac{U^{S}\left(\eta_{d}, \eta\right)}{\hat{\phi}_{O}(1)^{n}}$. We can deduce from Lemma 3 that the former limit is finite (in fact, zero) whereas the latter one tends to infinity since $\frac{\hat{\phi}_{S, \infty}(x)}{\hat{\phi}_{O}(1)}>1$ for $x$ in an interval $(1-\epsilon, 1]$ by Lemma 2 and item 3 of Proposition 2.

It only remains to be shown that $\frac{U^{S}\left(\eta_{d}, \eta\right)-U^{S}(\eta, \eta)}{\eta_{d}-\eta}>0$ under the T-information assumption for $n$ large enough. We shall use again a limit argument. Note from the arguments at the beginning of the proof that $U^{S}(\eta, \eta)=\int_{0}^{1}(1-x) x^{n} \frac{\partial E\left[T_{d}+Q_{d} \mid X_{d}^{\eta_{d}}=x\right]}{\partial x} \mathrm{~d} x$, consequently $\lim _{n \rightarrow \infty} U^{S}(\eta, \eta)=0$. On the other hand, Eq. (17) implies that $\lim _{n \rightarrow \infty} U^{S}\left(\eta_{d}, \eta\right)>0$ for $\eta_{d}>\eta$. To see why note that $\phi_{S, \infty}^{-1}(1)<1$ by Lemma 2 and item 3 of Proposition 2. Finally, $\lim _{n \rightarrow \infty} \frac{U^{S}\left(\eta_{d}, \eta\right)}{\hat{\phi}_{S, n}(1)^{n}}$ is finite (in fact, zero) whereas $\lim _{n \rightarrow \infty} \frac{U^{S}(\eta, \eta)}{\hat{\phi}_{S, n}(1)^{n}}=\infty$ if $\eta_{d}<\eta$ because Lemma 2 and item 3 of Proposition 2 imply that $\frac{x}{\hat{\phi}_{S, \infty}(1)}>1$ for $x$ in an interval $(1-\epsilon, 1]$.

Proof of Proposition 4. We start with the Q-information assumption. A necessary condition for $\eta \in(0,1)$ to be a symmetric Nash equilibrium is that $\lim _{\eta_{d} \rightarrow \eta} \frac{\partial U^{a}}{\partial \eta_{d}}\left(\eta_{d}, \eta\right)=C^{\prime}(\eta), a \in\{O, S\}$. This equation has a unique solution $\eta_{*}^{a} \in(0,1)$ for $\alpha$ sufficiently large since $C^{\prime}(\eta)=\alpha \eta$, and $\lim _{\eta_{d} \rightarrow \eta} \frac{\partial U^{a}}{\partial \eta_{d}}\left(\eta_{d}, \eta\right)>0$ and is continuous in $\eta$. A sufficient condition for $\eta_{*}^{a}$ to be an equilibrium is that $\frac{\Delta U^{a}}{\Delta \eta}\left(\eta_{d}, \eta_{*}^{a}\right)-\frac{C\left(\eta_{d}\right)-C\left(\eta_{*}^{a}\right)}{\eta_{d}-\eta_{*}^{a}}<0$ if and only if $\eta_{d}>\eta_{*}^{a}$. This is the case for $\alpha$ sufficiently large since $\frac{C\left(\eta_{d}\right)-C\left(\eta_{*}^{a}\right)}{\eta_{d}-\eta_{*}^{a}}=\frac{\alpha}{2}\left(\eta_{d}+\eta_{*}^{a}\right), \frac{\Delta U^{a}}{\Delta \eta}\left(\eta_{d}, \eta_{*}^{a}\right)>0$ and $\frac{\Delta U^{a}}{\Delta \eta}\left(\eta_{d}, \eta_{*}^{a}\right)$ is continuous, and $\lim _{\eta_{d} \rightarrow \eta_{*}^{a}}\left(\frac{\Delta U^{a}}{\Delta \eta}\left(\eta_{d}, \eta_{*}^{a}\right)-\frac{C\left(\eta_{d}\right)-C\left(\eta_{*}^{a}\right)}{\eta_{d}-\eta_{*}^{a}}\right)=0$ by definition of $\eta_{*}^{a}$. The fact that $\frac{\partial U^{a}}{\partial \eta_{d}}\left(\eta_{*}^{a}, \eta_{*}^{a}\right)=C^{\prime}\left(\eta_{*}^{a}\right), C^{\prime \prime}(\eta)>0$ and the limit version of the first item of Proposition 3 imply that $\eta_{*}^{S}>\eta_{*}^{O}$. The proof of the second item is similar but we need to start choosing an $n$ large enough so that the results in Proposition 3 apply for any $\left\{\left(\eta_{d}, \eta\right) \in[0,1]^{2}\right\}$. 
Proof of Lemma 4. For standard-symmetric auctions, the level of allocative efficiency corresponds to $E\left[\max \left\{E\left[T_{i} \mid X_{i}^{\eta}\right]\right\}_{i \in \mathcal{I}}\right]+n E\left[Q_{i}\right]$. This expression is constant with respect to $\eta$ under the Q-information assumption because $E\left[T_{i} \mid X_{i}^{\eta}\right]$ is constant in $\eta$. This also means that ax ante efficiency is decreasing since increasing $\eta$ is costly. Next note that by symmetry of the $X_{i}^{\eta}$,s, and monotonicity of $E\left[T_{i} \mid X_{i}^{\eta}=x\right]$ with respect to $x, E\left[\max \left\{E\left[T_{i} \mid X_{i}^{\eta}\right]\right\}_{i \in \mathcal{I}}\right]=E\left[E\left[T_{i} \mid X_{i}^{\eta} \geqslant\right.\right.$ $\left.\left.\max \left\{X_{j}^{\eta}\right\}_{j \neq i}\right]\right]$ which is increasing in $\eta$ under the T-information assumption since $E\left[T_{i} \mid X_{i}^{\eta} \geqslant x\right]$ is increasing in $\eta$ for any $x$ by Lemma 8 in Appendix A.1.

Proof of Lemma 5. Leaving aside information acquisition costs, the auctioneer's expected revenue is equal to the difference between the expected social surplus, i.e.

$$
\int_{0}^{1} E\left[T_{i} \mid X_{i}^{\eta}=x\right] \mathrm{d} x^{n}+n E\left[Q_{i}\right],
$$

and the sum of all bidders' expected utilities, i.e.

$$
\int_{0}^{1}(1-x) \frac{\partial E\left[T_{i}+Q_{i} \mid X_{i}^{\eta}=x\right]}{\partial x} \mathrm{~d} x^{n} .
$$

The latter one is an application of Lemma 3 for $\phi(x)=x$.

The first result follows since under the Q-information assumption, Eq. (18) is constant in $\eta$, whereas Eq. (19) is increasing in $\eta$.

To prove the second result, we can combine Eqs. (18) and (19) to show after some algebra that the auctioneer's expected revenue is equal to:

$$
\begin{gathered}
\int_{0}^{1}\left(E\left[T_{i} \mid X_{i}^{\eta}=x\right]-(1-x) \frac{\partial E\left[T_{i} \mid X_{i}^{\eta}=x\right]}{\partial x}\right) \mathrm{d} x^{n} \\
+n E\left[Q_{i}\right]-\int_{0}^{1}(1-x) \frac{\partial E\left[Q_{i} \mid X_{i}^{\eta}=x\right]}{\partial x} \mathrm{~d} x^{n} .
\end{gathered}
$$

The second integral is constant with respect to $\eta$ under the T-information assumption, whereas the integrand of the first integral is increasing in $\eta$ for values of $x$ close to 1 . The latter because $E\left[T_{i} \mid X_{i}^{\eta}=x\right]$ is strictly increasing in $\eta$ for $x=1$ by Lemma 8 in Appendix A. 1 and by continuity for any $x$ close to 1 . As a consequence, the above expression is increasing in $\eta$ for $n$ large enough.

\section{A.3. Conditions [A.1]-[A.4] for Example A}

\section{[A.1]-[A.3]}

We start by showing that [A.1]-[A.3] hold true for $a=S$ in Example A and under the Qinformation assumption. To simplify notation, we denote ${ }^{23} \bar{q} \equiv \eta_{T}(\eta), \eta^{Q} \equiv \eta_{Q}(\eta) / 2$ and $\eta_{d}^{Q} \equiv$

23 Note that to show differentiability and continuity with respect to $\eta$ is the same as to show differentiability and continuity with respect to $\eta^{Q}$ since $\eta_{Q}$ is a differentiable function and $\eta_{T}$ is constant under the Q-information assumption. 
$\eta_{Q}\left(\eta_{d}\right) / 2$. From Lemma 3, we can deduce that it is sufficient to show that the function $\phi_{S, n}$ in Proposition 2, which is explicitly built in the proof of Lemma 13 and that we shall denote in this appendix by ${ }^{24} \phi_{S, n}\left(x, \eta_{d}^{Q}, \eta^{Q}\right)$ is:

(i) continuous in $\left(\eta^{Q}, \eta_{d}^{Q}\right)$;

(ii) its limit:

$$
\lim _{\eta_{d}^{Q} \rightarrow \eta^{Q}} \frac{\phi_{S, n}\left(x, \eta_{d}^{Q}, \eta^{Q}\right)-\phi_{S, n}\left(x, \eta^{Q}, \eta^{Q}\right)}{\eta_{d}^{Q}-\eta^{Q}}
$$

exists and is continuous in $\eta^{Q}$ for $x<1$; and

(iii) the above limit is strictly positive for $x>0$ (recall that $\phi_{O}(x)=x$ and hence invariant with respect to $\left.\left(\eta_{d}^{Q}, \eta^{Q}\right)\right)$.

Note first the following characterization.

Lemma 14. If $\eta_{d}^{Q}<\eta^{Q}$ then $\phi_{S, n}\left(x, \eta_{d}^{Q}, \eta^{Q}\right)=x \cdot k\left(\eta_{d}^{Q}, \eta^{Q}\right)$, and if $\eta_{d}^{Q}>\eta^{Q}$ then $\phi_{S, n}\left(x, \eta_{d}^{Q}\right.$, $\left.\eta^{Q}\right) \in\left[x, x \cdot k\left(\eta_{d}^{Q}, \eta^{Q}\right)\right]($ strictly if $x>0)$, where $k\left(\eta_{d}^{Q}, \eta^{Q}\right) \equiv \frac{\eta_{d}^{Q}+\bar{\eta}+\frac{\bar{\eta}}{n-2}}{\eta^{Q}+\bar{\eta}+\frac{\bar{\eta}}{n-2}}$.

Proof. First note that in Example A,

$$
\Phi\left(x, \phi, \eta_{d}^{Q}, \eta^{Q}\right)=\frac{\phi}{x(n-2)} \cdot \frac{\bar{\eta} \phi+(1-\bar{\eta}) 1 / 2-\bar{\eta} x-(1-\bar{\eta}) 1 / 2}{\bar{\eta} x+(1-\bar{\eta}) 1 / 2-\bar{\eta} \phi-(1-\bar{\eta}) 1 / 2+\eta_{d}^{Q} x-\eta^{Q} \phi},
$$

which after some simple algebra becomes

$$
\Phi\left(x, \phi, \eta_{d}^{Q}, \eta^{Q}\right)=\frac{\frac{\bar{\eta}}{n-2}}{\eta^{Q}+\bar{\eta}} \cdot \frac{\phi}{x} \cdot \frac{\phi-x}{\alpha\left(\eta_{d}^{Q}, \eta^{Q}\right) x-\phi}=\frac{\frac{\bar{\eta}}{n-2}}{\eta^{Q}+\bar{\eta}} \cdot \Theta\left(\phi / x, \alpha\left(\eta_{d}^{Q}, \eta^{Q}\right)\right),
$$

where $\alpha\left(\eta_{d}^{Q}, \eta^{Q}\right)=\frac{\eta_{d}^{Q}+\bar{\eta}}{\eta^{Q}+\bar{\eta}}$ and $\Theta(y, a) \equiv \frac{y(1-y)}{y-a}$. Note also that $\phi_{O}(x)=x$ and $\phi_{*}(x)=$ $\alpha\left(\eta_{d}^{Q}, \eta^{Q}\right) \cdot x$, and

$$
\Phi\left(x, k\left(\eta_{d}^{Q}, \eta^{Q}\right) \cdot x, \eta_{d}^{Q}, \eta^{Q}\right)=k\left(\eta_{d}^{Q}, \eta^{Q}\right),
$$

and thus $x \cdot k\left(\eta_{d}^{Q}, \eta^{Q}\right)$ is solution to our differential equation.

Consider first the case $\eta_{d}^{Q}<\eta^{Q}$, then $k\left(\eta_{d}^{Q}, \eta^{Q}\right) \in\left(\alpha\left(\eta_{d}^{Q}, \eta^{Q}\right), 1\right)$ and $\phi_{S, n}\left(x, \eta_{d}^{Q}, \eta^{Q}\right) \in$ $\left[\alpha\left(\eta_{d}^{Q}, \eta^{Q}\right), x\right]$. To see why the function $x \cdot k\left(\eta_{d}^{Q}, \eta^{Q}\right)$ is the solution defined in Lemma 13, note that any other solution that passes by a point above $x \cdot k\left(\eta_{d}^{Q}, \eta^{Q}\right)$ does not converge to $(0,0)$ as required. The reason is that any solution to our differential equation $\phi$ satisfies, $\phi^{\prime}(x)=$ $\Phi\left(x, \phi(x), \eta_{d}^{Q}, \eta\right)$ and $\Phi\left(x, \phi, \eta_{d}^{Q}, \eta\right)<k\left(\eta_{d}^{Q}, \eta\right)$ when $\phi>x \cdot k\left(\eta_{d}^{Q}, \eta^{Q}\right)$. The latter can be deduced from Eq. (21) since $\Theta(y, a)$ is decreasing ${ }^{25}$ in $y$ for $y>a$ and $a \in(0,1)$.

24 With some abuse of notation, we make explicit that $\phi_{S, n}$ and $\Phi$ are functions of $\eta_{d}^{Q}$ and $\eta Q$.

25 Note that $\frac{\partial \Theta(y, a)}{\partial y}=-\frac{(y-a)^{2}+a(1-a)}{(y-a)^{2}}$. 
Consider next the case $\eta_{d}^{Q}>\eta^{Q}$, then $k\left(\eta_{d}^{Q}, \eta^{Q}\right) \in\left(1, \alpha\left(\eta_{d}^{Q}, \eta^{Q}\right)\right)$ and $\phi_{S, n}\left(x, \eta_{d}^{Q}, \eta^{Q}\right) \in$ $\left[x, \alpha\left(\eta_{d}^{Q}, \eta^{Q}\right) x\right]$ since $\phi_{O}(x)=x$ and $\phi_{*}(x)=\alpha\left(\eta_{d}^{Q}, \eta^{Q}\right) x$. In this case, the lemma follows because $\phi_{S, n}\left(1, \eta_{d}^{Q}, \eta^{Q}\right)=1$ by the condition in Lemma $12, x \cdot k\left(\eta_{d}^{Q}, \eta^{Q}\right)$ is a solution to our differential equation and Step 2 in the proof of Lemma 13 implies that solutions do not cross.

Note that (i) at $\eta_{d}^{Q}=\eta^{Q}$ is straightforward since $\phi_{*}(x)=\phi_{O}(x)=x$, both $\phi_{*}$ and $\phi_{O}$ vary continuously in $\left(\eta_{d}^{Q}, \eta^{Q}\right)$ and $\phi_{S, n}$ is between $\phi_{*}(x)$ and $\phi_{O}(x)$. To show (i) for $\eta_{d}^{Q}<\eta^{Q}$ is also direct from Lemma 14. Consider next the case $\eta_{d}^{Q}>\eta^{Q}$. Then, $k\left(\eta_{d}^{Q}, \eta^{Q}\right)<\alpha\left(\eta_{d}^{Q}, \eta^{Q}\right)$ and by continuity, there exists a $\delta>0$ such that $k\left(\tilde{\eta}_{d}^{Q}, \tilde{\eta}^{Q}\right)<\alpha\left(\tilde{\eta}_{d}^{Q}, \tilde{\eta}^{Q}\right)$, if $\left|\left(\eta_{d}^{Q}, \eta^{Q}\right)-\left(\tilde{\eta}_{d}^{Q}, \tilde{\eta}^{Q}\right)\right| \leqslant \delta$. Denote by $\bar{k}\left(\eta_{d}^{Q}, \eta^{Q}\right)$ the maximum of $k\left(\tilde{\eta}_{d}^{Q}, \tilde{\eta}^{Q}\right)$ for $\left|\left(\eta_{d}^{Q}, \eta^{Q}\right)-\left(\tilde{\eta}_{d}^{Q}, \tilde{\eta}^{Q}\right)\right| \leqslant \delta$ and note first that $\Phi$ is continuously differentiable in the compact set:

$$
\left\{\left(x, \phi, \tilde{\eta}_{d}^{Q}, \tilde{\eta}^{Q}\right): x \geqslant \epsilon, \phi \in\left[x, \bar{k}\left(\eta_{d}^{Q}, \eta^{Q}\right) x\right],\left|\left(\eta_{d}^{Q}, \eta^{Q}\right)-\left(\tilde{\eta}_{d}^{Q}, \tilde{\eta}^{Q}\right)\right| \leqslant \delta\right\},
$$

for any $\epsilon>0$ and thus it satisfies a Lipschitz condition uniformly on the set. Next note that $\phi_{S, n}\left(x, \tilde{\eta}_{d}^{Q}, \tilde{\eta}^{Q}\right) \in\left[x, \bar{k}\left(\eta_{d}^{Q}, \eta^{Q}\right) x\right]$ if $\left|\left(\eta_{d}^{Q}, \eta^{Q}\right)-\left(\tilde{\eta}_{d}^{Q}, \tilde{\eta}^{Q}\right)\right| \leqslant \delta$ by Lemma 14 and the definition of $\bar{k}$. Consequently, our solution $\phi_{S, n}\left(x, \eta_{d}^{Q}, \eta^{Q}\right)$ is also continuous for $\eta_{d}^{Q}<\eta^{Q}$, and $x \in[\epsilon, 1]$ by application of Coddington and Levinson (1984, Theorem 7.4, p. 29). Note that continuity also holds true for any $x \in[0,1]$ since $\phi_{S, n}\left(0, \eta_{d}^{Q}, \eta^{Q}\right) \in\left[\phi_{O}(0), \phi_{*}(0)\right]=\{0\}$.

To prove (ii) and (iii), note that by Lemma 14 and after some algebra,

$$
\lim _{\substack{Q \\ \eta_{d}^{Q} \rightarrow \eta \\ \eta_{d}^{Q} \leqslant \eta}} \frac{\phi_{S, n}\left(x, \eta_{d}^{Q}, \eta^{Q}\right)-\phi_{S, n}\left(x, \eta^{Q}, \eta^{Q}\right)}{\eta_{d}^{Q}-\eta^{Q}}=\frac{x}{\eta^{Q}+\bar{\eta}+\frac{\bar{\eta}}{n-2}} .
$$

We finish by showing that for any $x<1$,

$$
\lim _{\substack{\eta_{d}^{Q} \rightarrow \eta \\ \eta_{d}^{Q}>\eta}} \frac{\phi_{S, n}\left(x, \eta_{d}^{Q}, \eta^{Q}\right)-\phi_{S, n}\left(x, \eta^{Q}, \eta^{Q}\right)}{\eta_{d}^{Q}-\eta^{Q}}=\frac{x}{\eta^{Q}+\bar{\eta}+\frac{\bar{\eta}}{n-2}} .
$$

Since $\phi_{S, n}\left(x, \eta_{d}^{Q}, \eta^{Q}\right)$ is solution to our problem, it satisfies ${ }^{26}$ :

$$
\phi_{S, n}^{\prime}\left(x, \eta_{d}^{Q}, \eta^{Q}\right)=\Phi\left(x, \phi_{S, n}\left(x, \eta_{d}^{Q}, \eta^{Q}\right), \eta_{d}^{Q}, \eta^{Q}\right),
$$

which from Eq. (20) and after some algebra becomes

$$
\frac{\phi_{S, n}\left(x, \eta_{d}^{Q}, \eta^{Q}\right)-x}{\eta_{d}^{Q}-\eta^{Q}}=\frac{x}{\eta^{Q}+\bar{\eta}+\frac{\bar{\eta}}{n-2} \cdot \frac{\phi_{S, n}\left(x, \eta_{d}^{Q}, \eta^{Q}\right)}{x \phi_{S, n}^{\prime}\left(x, \eta_{d}^{Q}, \eta^{Q}\right)}} .
$$

Since $\lim _{\substack{\eta_{d}^{Q} \rightarrow \eta Q \\ \eta_{d}^{Q}>\eta}} \phi_{S, n}\left(x, \eta_{d}^{Q}, \eta^{Q}\right)=x$, see Lemma 14, it is sufficient to show that:

$$
\lim _{\substack{\eta_{d}^{Q} \rightarrow \eta Q \\ \eta_{d}^{Q}>\eta}} \phi_{S, n}^{\prime}\left(x, \eta_{d}^{Q}, \eta^{Q}\right)=1,
$$

26 Where $\phi_{S, n}^{\prime}\left(x, \eta^{Q}, \eta^{Q}\right)$ denotes the differential with respect to $x$. 
for $x<1$. To prove so, note that $\phi_{S, n}^{\prime}\left(x, \eta_{d}^{Q}, \eta^{Q}\right) \leqslant k\left(\eta_{d}^{Q}, \eta^{Q}\right)$ if $\eta_{d}^{Q}>\eta^{Q}$. This can be shown applying Lemma 14 and using that $\Phi\left(x, \phi, \eta_{d}^{Q}, \eta\right) \leqslant k\left(\eta_{d}^{Q}, \eta\right)$ for $\phi \leqslant x \cdot k\left(\eta_{d}^{Q}, \eta^{Q}\right)$. The latter because $x \cdot k\left(\eta_{d}^{Q}, \eta^{Q}\right)$ solves our differential equation and the monotonicity of $\Phi\left(x, \phi, \eta_{d}^{Q}, \eta\right)$ with respect to $\phi$ when $\phi \in\left[x, \alpha\left(\eta_{d}^{Q}, \eta^{Q}\right) x\right]$, see Eq. (20). Hence, $\limsup _{\substack{\eta_{d}^{Q} \rightarrow \eta Q \\ \eta_{d}^{Q}>\eta}} \phi_{S, n}^{\prime}\left(x, \eta_{d}^{Q}\right.$, $\left.\eta^{Q}\right) \leqslant 1$ since $k\left(\eta^{Q}, \eta^{Q}\right)=1$. To complete the proof, we show that,

$\lim \inf _{\substack { Q \\ \begin{subarray}{c}{d \\ \eta_{d}^{Q}>\eta{ Q \\ \begin{subarray} { c } { d \\ \eta _ { d } ^ { Q } > \eta } }\end{subarray}} \phi_{S, n}^{\prime}\left(x, \eta_{d}^{Q}, \eta^{Q}\right) \geqslant 1$,

for $x<1$. Suppose not. Then there must exists a sequence of $\eta_{d}^{Q}$ converging to $\eta^{Q}$ such that $\eta_{d}^{Q}>\eta^{Q}$ and $\phi_{S, n}^{\prime}\left(x, \eta_{d}^{Q}, \eta^{Q}\right)<1$ along the sequence. Note that $\phi_{S, n}^{\prime}\left(x, \eta_{d}^{Q}, \eta^{Q}\right)<1$ implies that $\phi_{S, n}^{\prime}\left(x, \eta_{d}^{Q}, \eta^{Q}\right)$ is decreasing in $x$. This can be proved by differentiating both sides of:

$$
\phi_{S, n}^{\prime}\left(x, \eta_{d}^{Q}, \eta^{Q}\right)=\Phi\left(x, \phi_{S, n}\left(x, \eta_{d}^{Q}, \eta^{Q}\right), \eta_{d}^{Q}, \eta^{Q}\right),
$$

with respect to $x$ and using that $\phi_{S, n}\left(x, \eta_{d}^{Q}, \eta^{Q}\right) \in\left[x, \alpha\left(\eta_{d}^{Q}, \eta^{Q}\right) x\right]$ for $\eta_{d}^{Q}>\eta^{Q}$. Hence:

$$
\begin{aligned}
& \phi_{S, n}\left(x, \eta_{d}^{Q}, \eta^{Q}\right)+(1-x) \phi_{S, n}^{\prime}\left(x, \eta_{d}^{Q}, \eta^{Q}\right) \\
& \quad=\phi_{S, n}\left(x, \eta_{d}^{Q}, \eta^{Q}\right)+\int_{x}^{1} \phi_{S, n}^{\prime}\left(x, \eta_{d}^{Q}, \eta^{Q}\right) \mathrm{d} \tilde{x} \\
& \quad \geqslant \phi_{S, n}\left(x, \eta_{d}^{Q}, \eta^{Q}\right)+\int_{x}^{1} \phi_{S, n}^{\prime}\left(\tilde{x}, \eta_{d}^{Q}, \eta^{Q}\right) \mathrm{d} \tilde{x}=\phi_{S, n}\left(1, \eta_{d}^{Q}, \eta^{Q}\right)=1 .
\end{aligned}
$$

We can thus conclude that

$$
\phi_{S, n}^{\prime}\left(x, \eta_{d}^{Q}, \eta^{Q}\right) \geqslant \frac{1-\phi_{S, n}\left(x, \eta_{d}^{Q}, \eta^{Q}\right)}{1-x} .
$$

Hence, $\lim _{\eta_{d}^{Q} \rightarrow \eta^{Q}} \phi_{S, n}\left(x, \eta_{d}^{Q}, \eta^{Q}\right)=x$ means that

$$
\lim \inf _{\substack{\eta \\ \eta_{d} \rightarrow \eta \\ \eta_{d}^{Q}>\eta}} \phi_{S, n}^{\prime}\left(x, \eta_{d}^{Q}, \eta^{Q}\right) \geqslant 1
$$

which contradicts our starting assumption.

\section{[A.4]}

We show next that [A.4] holds true for our Example A when $\eta_{Q}(\eta)=0.2$ and $\eta_{T}(\eta) \in$ $[0.2,0.4]$. To simplify notation, we denote $\bar{\eta} \equiv \frac{\eta_{Q}(\eta)}{2}$ (hence, $\left.\bar{\eta}=0.1\right), \eta^{T} \equiv \eta_{T}(\eta)$ and $\eta_{d}^{T} \equiv$ $\eta_{T}\left(\eta_{d}\right)$. In this case, we have that:

$$
\Phi(x, \phi)=\frac{\phi}{(n-2) x} \cdot \frac{\eta^{T}(\phi-1 / 2)-\eta_{d}^{T}(x-1 / 2)}{\left(\eta_{d}^{T}+\bar{\eta}\right)(x-1 / 2)-\left(\eta^{T}+\bar{\eta}\right)(\phi-1 / 2)},
$$

$\phi_{O}(x)=\beta_{O}(x-1 / 2)+1 / 2$, where $\beta_{O} \equiv \frac{\eta_{d}^{T}}{\eta^{T}}$, and $\phi_{*}(x)=\beta_{*}(x-1 / 2)+1 / 2$, where $\beta_{*} \equiv \frac{\eta_{d}^{T}+\bar{\eta}}{\eta^{T}+\bar{\eta}}$.

In what follows we deduce some bounds, Eqs. (22)-(27), to prove [A.4]. 
- Case $\eta_{d}^{T}>\eta^{T}\left(\eta_{d}>\eta\right)$

Note that if $x \leqslant 1 / 2$, then $\hat{\phi}_{O}(x) \leqslant \hat{\phi}_{*}(x)$ and hence $\hat{\phi}_{S, n}(x) \in\left[\hat{\phi}_{O}(x), \hat{\phi}_{*}(x)\right]$, by item 1 of Proposition 2, whereas if $x \geqslant 1 / 2$, then $\hat{\phi}_{*}(x) \leqslant \hat{\phi}_{O}(x)$, and hence $\hat{\phi}_{S, n}(x) \in$ $\left[\hat{\phi}_{*}(x), \hat{\phi}_{O}(x)\right]$. Thus, we can deduce from Lemma 3 our first bound,

$$
\begin{aligned}
\frac{\Delta U^{S}}{\Delta \eta}\left(\eta_{d}, \eta\right) \geqslant & \frac{\left(\eta_{d}^{T}+2 \bar{\eta}\right)}{\eta_{d}-\eta}\left(\int_{0}^{1 / 2}(1-x)\left(\hat{\phi}_{O}(x)^{n-1}-x^{n-1}\right) \mathrm{d} x\right. \\
& \left.+\int_{1 / 2}^{1}(1-x)\left(\hat{\phi}_{*}(x)^{n-1}-x^{n-1}\right) \mathrm{d} x\right) .
\end{aligned}
$$

Next, let $\phi_{U}(x) \equiv \beta_{U}(x-1 / 2)+1 / 2$, where $\beta_{U}=\frac{\eta_{d}^{T}+(n-2)\left(\eta_{d}^{T}+\bar{\eta}\right)}{\eta^{T}+(n-2)\left(\eta^{T}+\bar{\eta}\right)}$, and by $\hat{\phi}_{U}$ its extension. Note that $\hat{\phi}_{U}(x) \in\left[\hat{\phi}_{*}(x), \hat{\phi}_{O}(x)\right]$, if $x \geqslant 1 / 2$ since $\beta_{U} \in\left(\beta_{*}, \beta_{O}\right)$. Moreover, $\Phi\left(x, \phi_{U}(x)\right)<\beta_{U}$ for $x \in(1 / 2,1]$ since $\frac{\phi_{U}(x)}{x}<\beta_{U}$ and it may be shown after some algebra that

$$
\frac{\eta^{T}\left(\phi_{U}(x)-1 / 2\right)-\eta_{d}^{T}(x-1 / 2)}{(n-2)\left(\left(\eta_{d}^{T}+\bar{\eta}\right)(x-1 / 2)-\left(\eta^{T}+\bar{\eta}\right)\left(\phi_{U}(x)-1 / 2\right)\right)}=1
$$

for $x>1 / 2$. We can also use a similar argument to show that

$$
\limsup _{\substack{(x, \phi) \rightarrow(1 / 2,1 / 2) \\ x \geqslant 1 / 2, \phi \in\left[\phi_{U}(x), \phi_{O}(x)\right]}} \Phi(x, \phi)=1<\beta_{U} .
$$

Consequently, $\hat{\phi}_{S, n}(x) \leqslant \phi_{U}(x)$ for $x \in[1 / 2,1]$, whereas from the arguments above, we know that $\hat{\phi}_{S, n}(x) \leqslant \phi_{O}(x)$ for $x \in[0,1 / 2]$. We can thus derive our second bound,

$$
\begin{aligned}
\frac{\Delta U^{O}}{\Delta \eta}\left(\eta_{d}, \eta\right)-\frac{\Delta U^{S}}{\Delta \eta}\left(\eta_{d}, \eta\right) \geqslant & \frac{\eta_{d}^{T}+2 \bar{\eta}}{\eta_{d}-\eta}\left(\int_{0}^{1 / 2}(1-x)\left(\hat{\phi}_{O}(x)^{n-1}-\hat{\phi}_{*}(x)^{n-1}\right) \mathrm{d} x\right. \\
& \left.+\int_{1 / 2}^{1}(1-x)\left(\hat{\phi}_{O}(x)^{n-1}-\hat{\phi}_{U}(x)^{n-1}\right) \mathrm{d} x\right) .
\end{aligned}
$$

- Case $\eta_{d}^{T}<\eta^{T}\left(\eta_{d}<\eta\right)$

By a similar argument as above, we have that $\hat{\phi}_{S, n}(x) \in\left[\hat{\phi}_{O}(x), \hat{\phi}_{*}(x)\right]$ if $x \leqslant 1 / 2$, and $\hat{\phi}_{S, n}(x) \in\left[\hat{\phi}_{*}(x), \hat{\phi}_{O}(x)\right]$ if $x \geqslant 1 / 2$. Thus, we get our third bound, ${ }^{27}$

$$
\begin{aligned}
\frac{\Delta U^{S}}{\Delta \eta}\left(\eta_{d}, \eta\right) \geqslant & \frac{\left(\eta_{d}^{T}+2 \bar{\eta}\right)}{\eta_{d}-\eta}\left(\int_{0}^{1 / 2}(1-x)\left(\hat{\phi}_{*}(x)^{n-1}-x^{n-1}\right) \mathrm{d} x\right. \\
& \left.+\int_{1 / 2}^{1}(1-x)\left(\hat{\phi}_{O}(x)^{n-1}-x^{n-1}\right) \mathrm{d} x\right) .
\end{aligned}
$$

27 Note that $\eta_{d}-\eta<0$ and thus we need an upper bound for $U^{S}\left(\eta_{d}, \eta\right)$ and hence for $\phi_{S, n}$. 
Next, let $\beta_{L} \equiv \frac{\eta_{d}^{T}+\frac{(n-2)}{2}\left(\eta_{d}^{T}+\bar{\eta}\right)}{\eta^{T}+\frac{(n-2)}{2}\left(\eta^{T}+\bar{\eta}\right)} \in\left(\beta_{O}, \beta_{*}\right), \phi_{L}(x) \equiv \beta_{L}(x-1 / 2)+1 / 2$ and $\hat{\phi}_{L}(x)$ its extension. First, note that $\Phi\left(x, \phi_{L}(x)\right)<\beta_{L}$ if $\eta_{d}^{T}<\eta^{T}$ and $x>1 / 2$. This is because $\frac{\phi_{L}(x)}{x}=\left(1+\frac{1-\beta_{L}}{2 x \beta_{L}}\right) \beta_{L}<2 \beta_{L}$ (since $\beta_{L}>\frac{\eta_{d}^{T}}{\eta^{T}} \geqslant 1 / 2$ for $\left.\eta_{d}^{T}, \eta^{T} \in[0.2,0.4]\right)$ and,

$$
\frac{\eta^{T}\left(\phi_{L}(x)-1 / 2\right)-\eta_{d}^{T}(x-1 / 2)}{(n-2)\left(\left(\eta_{d}^{T}+\bar{\eta}\right)(x-1 / 2)-\left(\eta^{T}+\bar{\eta}\right)\left(\phi_{L}(x)-1 / 2\right)\right)}=\frac{1}{2} .
$$

Second, note that $\phi_{S, n}(1)=\phi_{*}(1)>\phi_{L}(1)$ by item 2 of Proposition 2 and $\phi_{*}(1)>\phi_{O}(1)$. Putting together these two facts we have that $\phi_{S, n}(x) \geqslant \phi_{L}(x)$ for $x \in[1 / 2,1]$. As a consequence, we derive our fourth bound,

$$
\begin{aligned}
\frac{\Delta U^{O}}{\Delta \eta}\left(\eta_{d}, \eta\right)-\frac{\Delta U^{S}}{\Delta \eta}\left(\eta_{d}, \eta\right) \geqslant & \frac{\eta_{d}^{T}+2 \bar{\eta}}{\eta_{d}-\eta}\left(\int_{0}^{1 / 2}(1-x)\left(\hat{\phi}_{O}(x)^{n-1}-\hat{\phi}_{*}(x)^{n-1}\right) \mathrm{d} x\right. \\
& \left.+\int_{1 / 2}^{1}(1-x)\left(\hat{\phi}_{O}(x)^{n-1}-\hat{\phi}_{L}(x)^{n-1}\right) \mathrm{d} x\right) .
\end{aligned}
$$

- Case $\eta_{d}=\eta\left(\eta_{d}=\eta\right)$

If we compute the limit $\eta_{d} \rightarrow \eta$ in Eqs. (22) and (23), we get respectively, our last two bounds,

$$
\begin{aligned}
\lim _{\eta_{d} \rightarrow \eta} \frac{\Delta U^{S}}{\Delta \eta}\left(\eta_{d}, \eta\right) \geqslant & \left(\eta^{T}+2 \bar{\eta}\right)(n-1) \eta_{T}^{\prime}(\eta)\left(\int_{0}^{1 / 2}(1-x) x^{n-2} \cdot \frac{x-1 / 2}{\eta^{T}} \mathrm{~d} x\right. \\
& \left.+\int_{1 / 2}^{1}(1-x) x^{n-2} \cdot \frac{x-1 / 2}{\eta^{T}+\bar{\eta}} \mathrm{d} x\right)
\end{aligned}
$$

since $\lim _{\eta_{d} \rightarrow \eta} \hat{\phi}_{O}(x)=\lim _{\eta_{d} \rightarrow \eta} \hat{\phi}_{*}(x)=x$. And,

$$
\begin{aligned}
\lim _{\eta_{d} \rightarrow \eta}\left(\frac{\Delta U^{O}}{\Delta \eta}\left(\eta_{d}, \eta\right)-\frac{\Delta U^{S}}{\Delta \eta}\left(\eta_{d}, \eta\right)\right) \\
\geqslant\left(\eta^{T}+2 \bar{\eta}\right)(n-1) \eta_{T}^{\prime}(\eta)\left(\int_{0}^{1 / 2}(1-x) x^{n-2}(x-1 / 2) \cdot \frac{\bar{\eta}}{\eta^{T}\left(\eta^{T}+\bar{\eta}\right)} \mathrm{d} x\right. \\
\left.\quad+\int_{1 / 2}^{1}(1-x) x^{n-2}(x-1 / 2) \cdot \frac{\bar{\eta}(n-2)}{\eta^{T}\left(\eta^{T}+\left(\eta^{T}+\bar{\eta}\right)(n-2)\right)} \mathrm{d} x\right),
\end{aligned}
$$

since $\lim _{\eta_{d} \rightarrow \eta} \hat{\phi}_{O}(x)=\lim _{\eta_{d} \rightarrow \eta} \hat{\phi}_{*}(x)=\lim _{\eta_{d} \rightarrow \eta} \hat{\phi}_{U}(x)=x$.

Numerical computations show that Eqs. (22)-(27) are strictly positive for $n=5$ and $\eta_{d}^{T}, \eta^{T} \in$ $[0.2,0.4]$. 


\section{References}

Athey, S., Levin, J., 2001. The value of information in monotone decision problems. Discussion paper 98-24, MIT.

Bagnoli, M., Bergstrom, T., 2005. Log-concave probability and its applications. Econ. Theory 26, 445-469.

Bergemann, D., Välimäki, J., 2002. Information acquisition and efficient mechanism design. Econometrica 70, 10071033.

Bickel, P.J., Lehmann, E.L., 1976. Descriptive statistics for nonparametric models. III. Dispersion. Ann. Statist. 4, 11391158 .

Bikhchandani, S., Riley, J.G., 1991. Equilibria in open common value auctions. J. Econ. Theory 53, 101-130.

Bikhchandani, S., Haile, P.A., Riley, J.G., 2002. Symmetric separating equilibria in English auctions. Games Econ. Behav. 38, 19-27.

Boone, J., Goeree, J., 2005. Optimal privatization using qualifying auctions. Unpublished, University of Tilburg.

Coddington, E.A.E., Levinson, N., 1984. Theory of Ordinary Differential Equations, first ed. Krieger Publishing Company.

Compte, O., Jehiel, P., 2002. Auctions and information acquisition: Sealed bid or dynamic formats. Discussion paper. ELSE, University College London.

Engelbrecht-Wiggans, R., 2001. The effect of entry and information costs on oral versus sealed-bid auctions. Econ. Letters 70, 195-202.

Engelbrecht-Wiggans, R., Milgrom, P.R., Weber, R.J., 1983. Competitive bidding and proprietary information. J. Math. Econ. 11, 161-169.

Ganuza, J.-J., Penalva-Zuasti, J.S., 2006. Information transmission in private value auctions. Unpublished, University Pompeu Fabra.

Hagedorn, M., 2004. The value of information in auctions. Unpublished, University of Bonn.

Harstad, R.M., 1990. Alternative common-value auction procedures: Revenue comparisons with free entry. J. Polit. Economy 98 (2), 421-429.

Hausch, D.B., Li, L., 1993. A common value auction model with endogenous entry and information acquisition. Econ. Theory 3, 315-334.

Hernando-Veciana, A., 2004. Successful uninformed bidding. Games Econ. Behav. 48 (1), 29-53.

Hernando-Veciana, A., Tröge, M., 2005. The insider's curse. Discussion paper WP-AD 2005-08, IVIE.

Krishna, V., 2003. Asymmetric English auctions. J. Econ. Theory 112, 261-288.

Larson, N., 2004. Private value perturbations and informational advantage in common value auctions. Unpublished, available at SSRN: http://ssrn.com/abstract=665121.

Lee, T.K., 1985. Competition and information acquisition in first price auctions. Econ. Letters 18, $129-132$.

Lehmann, E.L., 1988. Comparing location experiments. Ann. Statist. 16 (2), 521-533.

Levin, D., Smith, J.L., 1994. Equilibrium in auctions with entry. Amer. Econ. Rev. 84 (3), 585-599.

Matthews, S., 1984. Information acquisition in discriminatory auctions. In: Bayesian Models in Economic Science. Elsevier Science.

McAfee, R.P., McMillan, J., 1987. Auctions with a stochastic number of bidders. J. Econ. Theory 43, 1-19.

Milgrom, P., 1981. Rational expectations, information acquisitions, and competitive bidding. Econometrica 49 (4), $921-$ 943.

Milgrom, P., Weber, R., 1982a. A theory of auctions and competitive bidding. Econometrica 50, 1089-1122.

Milgrom, P., Weber, R., 1982b. The value of information in a sealed-bid auction. J. Math. Econ. 10, $105-114$.

Myerson, R.B., 1981. Optimal auction design. Math. Operations Res. 6 (1), 58-73.

Parreiras, S., 2002. Common value auctions with asymmetrically informed bidders. Unpublished, University of North Carolina.

Persico, N., 2000. Information acquisition in auctions. Econometrica 68 (1), 135-148.

Pesendorfer, W., Swinkels, J.M., 1997. The loser's curse and information aggregation in common value auctions. Econometrica 65 (6), 1247-1281.

Rothschild, M., Stiglitz, J., 1970. Increasing risk: I. A definition. J. Econ. Theory 2 (3), 225-243.

Stegeman, M., 1996. Participation costs and efficient auctions. J. Econ. Theory 71, 228-259.

Vickrey, W., 1961. Counterspeculation, auctions, and competitive sealed tenders. J. Finance 16, 8-37.

Ye, L., 2007. Indicative bidding and a theory of two-stage auctions. Games Econ. Behav. 58, 181-207. 